\title{
The association of a La module with the PABP-interacting motif PAM2 is a recurrent evolutionary process that led to the neofunctionalization of La-related proteins
}

\author{
RÉMY MERRET, ${ }^{1,2,4}$ LUIGI MARTINO, ${ }^{3,4}$ CÉCILE BOUSQUET-ANTONELLI, ${ }^{1,2,4,5}$ SARA FNEICH, ${ }^{1,2}$ \\ JULIE DESCOMBIN, ${ }^{1,2}$ ÉLODIE BILLEY, ${ }^{1,2}$ MARIA R. CONTE, ${ }^{3,4,5}$ and JEAN-MARC DERAGON ${ }^{\mathbf{1}, 2,4}$ \\ ${ }^{1}$ Université de Perpignan Via Domitia, UMR5096 LGDP, 66860 Perpignan Cedex, France \\ ${ }^{2}$ CNRS, UMR5096 LGDP, 66860 Perpignan Cedex, France \\ ${ }^{3}$ Randall Division of Cell and Molecular Biophysics, King's College London, Guy's Campus, London SE1 1UL, United Kingdom
}

\begin{abstract}
La-related proteins (LARPs) are largely uncharacterized factors, well conserved throughout evolution. Recent reports on the function of human LARP4 and LARP6 suggest that these proteins fulfill key functions in mRNA metabolism and/or translation. We report here a detailed evolutionary history of the LARP4 and 6 families in eukaryotes. Genes coding for LARP4 and 6 were duplicated in the common ancestor of the vertebrate lineage, but one LARP6 gene was subsequently lost in the common ancestor of the eutherian lineage. The LARP6 gene was also independently duplicated several times in the vascular plant lineage. We observed that vertebrate LARP4 and plant LARP6 duplication events were correlated with the acquisition of a PABPinteracting motif 2 (PAM2) and with a significant reorganization of their RNA-binding modules. Using isothermal titration calorimetry (ITC) and immunoprecipitation methods, we show that the two plant PAM2-containing LARP6s (LARP6b and c) can, indeed, interact with the major plant poly(A)-binding protein (PAB2), while the third plant LARP6 (LARP6a) is unable to do so. We also analyzed the RNA-binding properties and the subcellular localizations of the two types of plant LARP6 proteins and found that they display nonredundant characteristics. As a whole, our results support a model in which the acquisition by LARP4 and LARP6 of a PAM2 allowed their targeting to mRNA 3' UTRs and led to their neofunctionalization.
\end{abstract}

Keywords: RNA-binding proteins; RRM; PAM2; PABP; LAM

\section{INTRODUCTION}

The La-motif (LAM) protein superfamily is composed of RNA-binding proteins conserved across eukaryotes (Bousquet-Antonelli and Deragon 2009). It comprises five distinct families: the genuine La family and the La-related protein (LARP) families 1, 4, 6, and 7. For most members of this superfamily, the LAM is followed by a more or less canonical RNA-recognition motif (named RRM or RRM-like) forming a functional RNA-binding unit named the La module (Bousquet-Antonelli and Deragon 2009; Bayfield et al. 2010).

The genuine La protein family has been the subject of numerous studies over the years, and their members are known to recognize the $3^{\prime} \mathrm{UUU}-\mathrm{OH}$ tail of several small

\footnotetext{
${ }^{4}$ These authors contributed equally to this work.

${ }^{5}$ Corresponding authors

E-mail cecile.antonelli@univ-perp.fr

E-mail sasi.conte@kcl.ac.uk

Article published online ahead of print. Article and publication date are at http://www.rnajournal.org/cgi/doi/10.1261/rna.035469.112.
}

RNA precursors (such as tRNAs, snoRNAs, and various snRNAs), ensuring their protection against exonucleases and facilitating their correct maturation and folding (Wolin and Cedervall 2002; Bayfield et al. 2010). This specific $3^{\prime} \mathrm{UUU}-\mathrm{OH}$ termini recognition is achieved through the synergic action of the LAM and the adjacent RRM1, that together form a uniquely tailored RNA-binding pocket, with several conserved amino acids in the LAM playing a crucial role in the interaction (Teplova et al. 2006; KotikKogan et al. 2008; Bayfield et al. 2010). In the cytoplasm, most genuine La proteins can also modulate the translation of a subset of mRNAs by binding to internal, often structured, RNA regions (Holcik and Sonenberg 2005; Svitkin et al. 2009; Martino et al. 2012). In this case, in addition to the LAM-RRM1 binding unit, a distal unconventional RRM2 is also involved (Martino et al. 2012). Despite this difference, the LAM/RRM1 surfaces implicated in binding internal RNA regions are likely to overlap, at least partially, with the regions responsible for terminal $3^{\prime} \mathrm{UUU}-\mathrm{OH}$ recognition (Martino et al. 2012). 
Of the LARP families studied for function so far, LARP7 is the best known. Vertebrate LARP7 binds specifically to 7SK snRNA, in part via its $3^{\prime} \mathrm{UUU}-\mathrm{OH}$, forming the core of a multicomponent ribonucleoprotein complex (the 7SK snRNP) involved in regulating Pol II transcription elongation by sequestering the positive elongation factor pTEF-b in an inactive state (Diribarne and Bensaude 2009; Bayfield et al. 2010; Xue et al. 2010). Also, Tetrahymena LARP7 (also named p65) binds to telomerase RNA and is essential for its accumulation in vivo (Witkin and Collins 2004; Singh et al. 2012). In contrast, LARP 1, 4, and 6 appear to be involved in mRNA metabolism and/or translation (Nykamp et al. 2008; Blagden et al. 2009; Burrows et al. 2010; Cai et al. 2010a,b; Schaffler et al. 2010; Yang et al. 2011). Recent works on human LARPs 4 and 6 have clearly established these proteins as important regulators of mRNA translation (Cai et al. 2010a,b; Schaffler et al. 2010; Yang et al. 2011). In human, two genes encode for the two LARP4 variants named HsLARP4a and HsLARP4b (HsLARP4b was previously designated HsLARP5). Although these two proteins clearly belong to the LARP4 family (Bousquet-Antonelli and Deragon 2009), they have acquired distinct characteristics through evolution. Human LARP4a and LARP4b are mostly homologous in their LAM and RRM-like domains, albeit presenting few differences for residues possibly involved in RNA recognition (Bayfield et al. 2010; Yang et al. 2011). Initial functional analysis of human LARP4a and LARP4b revealed common as well as distinct properties (Schaffler et al. 2010; Yang et al. 2011). In particular, both proteins were shown to bind directly to the poly(A)-binding protein (PABP) and to the receptor for activated C kinase (RACK1), a component of the $40 \mathrm{~S}$ ribosomal subunit. They also cosediment with polysomes in physiological conditions and localize to stress granules in stress situations. Finally, both appear to regulate translation, although, contrary to HsLARP4b, HsLARP4a does so at least in part by promoting mRNA stability (Schaffler et al. 2010; Yang et al. 2011). Moreover, whereas the RNA-binding properties of HsLARP4b have not yet been characterized, HsLARP4a bears a distinct preference for single-stranded poly(A) stretches containing $15 \mathrm{nt}$ (Yang et al. 2011). The ability of HsLARP4a to interact with poly(A) may constitute a necessary feature for its activity, because it appears to contribute to the stability of HsLARP4a/PABP and HsLARP4a/polysome associations (Yang et al. 2011).

LARP6 (formerly named Acheron) has also been studied mainly in human, where it is encoded by a single gene (Valavanis et al. 2007; Bousquet-Antonelli and Deragon 2009; Cai et al. 2010a,b). Human LARP6 regulates the synthesis of collagen $\alpha 1(\mathrm{I}), \alpha 2(\mathrm{I})$, and likely $\alpha 1(\mathrm{III})$ chains via a specific interaction with a unique stem-loop structure in the $5^{\prime}$ UTR of these mRNAs that includes the initiation codon and is conserved throughout vertebrates (Cai et al. 2010a,b). Recent work has shown that HsLARP6 coordinates the translation of the $\alpha 1$ (I) and $\alpha 2$ (I) collagen chains as well as their correct assembly, by colocalizing collagen mRNAs with intracellular nonmuscle myosin filaments whose association with polysomes controls the synthesis of heterotrimeric type I collagen (Cai et al. 2010a,b; Parsons et al. 2011).

Although LARP4 and LARP6 are largely represented across eukaryotes, where they appear to fulfill key cellular roles, the investigation of their biological function has been thus far restricted to the human proteins. In a first step to comprehend their functional role and relevance on a more global scale, we present here a detailed evolutionary history of the LARP4 and LARP6 families throughout eukaryotes. Moreover, through the analysis of some of the molecular interactions as well as the subcellular localization of the three Arabidopsis LARP6 proteins, we provide strong support to the view that the acquisition of a PABP-interacting motif 2 (PAM2) represents a key milestone in their neofunctionalization.

\section{RESULTS}

\section{LARP4 evolutionary history}

In a previous study, we observed that LARP4 is absent from plants and yeasts but present in some protists and in all animals tested, where it is encoded by a single gene, except for vertebrates displaying two LARP4 genes (BousquetAntonelli and Deragon 2009). To test the generality of these findings, we performed a more detailed analysis of the LARP4 family using 43 LARP4 proteins from 30 protist, invertebrate, and vertebrate species (see Supplemental Table S1 for a detailed list of the species and proteins used). The La modules (LAM-RRM) of all LARP4s were aligned and used to produce a phylogenetic tree (Fig. 1), confirming a single LARP4 gene in protist and invertebrate species, while the two genes coding for the two variants (LARP4a and LARP4b) were consistently found in vertebrates. Our phylogenetic analysis clearly establishes that LARP4a and LARP4b form two distinct lineages and should therefore be considered as two independent subfamilies (Fig. 1). It also supports the hypothesis of a gene duplication event that happened very early in the vertebrate lineage followed by the rapid divergence of the two duplicated copies, a situation often encountered in cases of combined sub and neofunctionalization (He and Zhang 2005). Only one vertebrate species used in this study (Dana rerio) has three instead of two LARP4 genes, possibly resulting from a supplemental and very recent duplication event of the LARP4a gene (Fig. 1).

The two human LARP4 proteins encompass an atypical PAM2 (PABP-interacting motif 2) sequence near their N-terminal region (Bayfield et al. 2010). PAM2 motifs are competent to bind to the MLLE peptide-binding domain (formerly known as PABPC) found at the $\mathrm{C}$ terminus of poly(A)-binding proteins (PABP) (Kozlov et al. 2010), and this interaction underlies the ability of PABP to recruit 


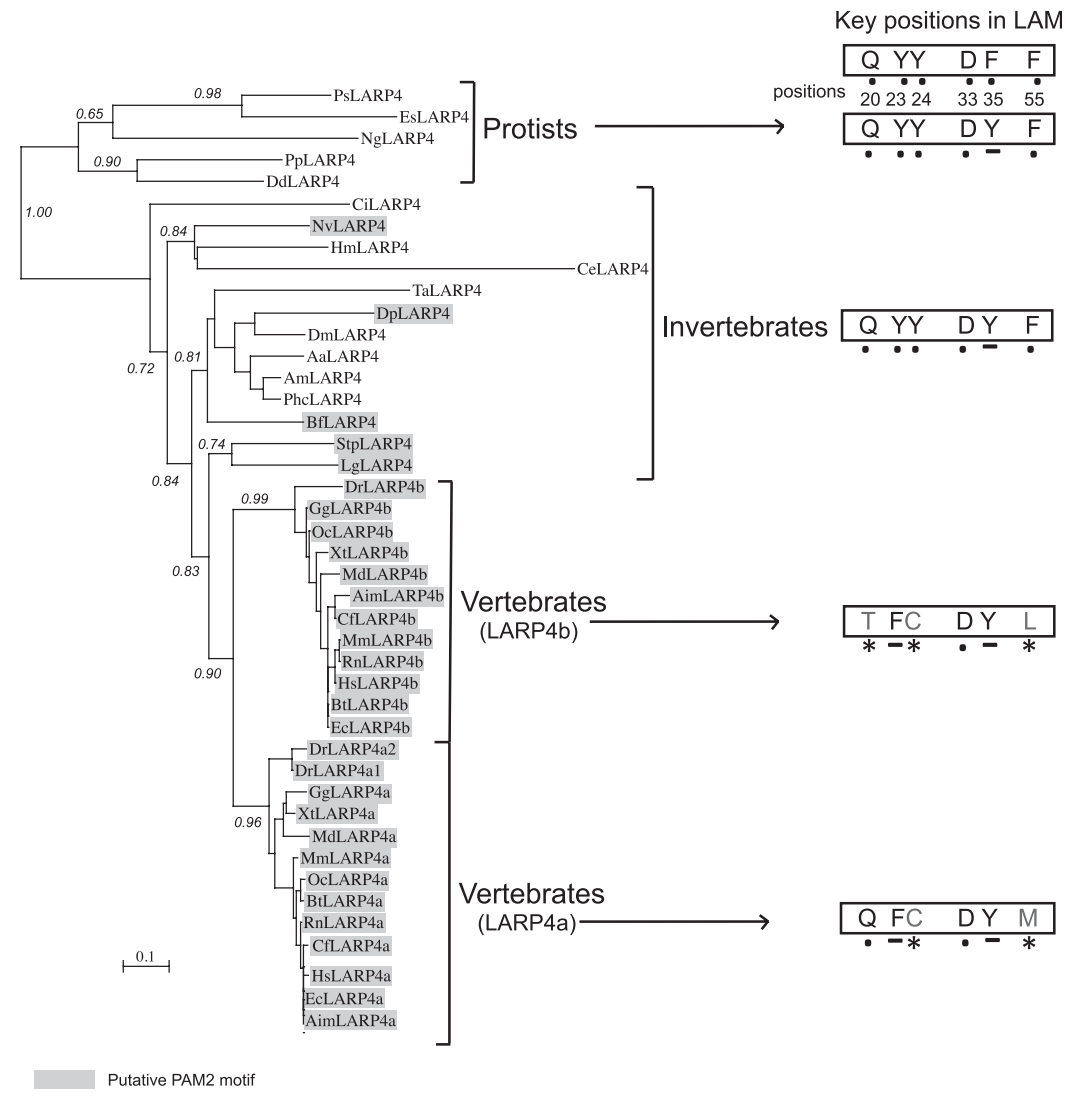

FIGURE 1. Detailed phylogenetic analysis of the LARP4 family. The phylogenetic tree was obtained using La module (LAM-RRM) sequences of 43 LARP4 proteins from protist, invertebrate, and vertebrate species (see Supplemental Table S1 for protein sequences). Selected informative statistical supports (approximate likelihood-ratio test [aLRT] data) are indicated. (Gray) Proteins presenting a putative PAM2 (see Fig. 2A). Key positions of the LAM, known to form the RNA-binding pocket in the context of the genuine La protein (Bayfield et al. 2010), are indicated on the right (dots indicate conserved amino acids, underlined amino acids are for possible conservative changes, and gray amino acids with an asterisk are for possible nonconservative changes). The numbering refers to the position of the amino acids in the human genuine LAM. Species codes are the following: (Ps) Phytophtora species; (Es) Ectocarpus siliculosus; (Ng) Naegleria gruberi; (Pp) Polysphondylium pallidum; (Dd) dictyostelium discoideum; (Ci) Ciona intestinalis; ( $\mathrm{Nv}$ ) Nematostella vectensis; $(\mathrm{Hm})$ Hydra magnipapillata; (Ce) Caenorhabditis elegans; (Ta) Trichoplax adhaerens; (Dp) Daphnia pullex; (Dm) Drosophila melanogaster; (Aa) Aedes aegypti; (Am) Apis mellifera; (Phc) Pediculus humanus corporis; (Bf) Branchiostoma floridae; (Stp) Strongylocentrotus purpuratus; (Lg) Lottia gigantae; (Dr) Danio rerio; (Gg) Gallus gallus; (Xt) Xenopus tropicalis; (Md) Monodelphis domestica; (Aim) Ailuropoda melanoleuca; (Cf) Canis familiaris; (Mm) Mus musculus; (Rn) Rattus norvegicus; (Hs) Homo sapiens; (Oc) Oryctolagus cuniculus; (Bt) Bos taurus; (Ec) Equus caballus.

various translation factors and proteins involved in translation regulation and mRNA stability (Craig et al. 1998; Khaleghpour et al. 2001a,b; Uchida et al. 2002; Funakoshi et al. 2007; Martineau et al. 2008). Although the PAM2-like sequence of both human LARP4 proteins is unusual, in that a phenylalanine reported to be invariable and essential for binding (Kozlov et al. 2010) is replaced by a tryptophan, the HsLARP4a PAM2 was shown to interact with the PABP MLLE domain in a similar fashion to the canonical PAM2 motif (Yang et al. 2011), suggesting that it could be functional in vivo.
A search for PAM2-like motifs in our collection of LARP4 proteins revealed positive hits in all vertebrates but none in protist proteins (Figs. 1, 2A). Unexpectedly, in invertebrate LARP4, the PAM2-like motif exhibits a patchy distribution with no clear relation to the evolutionary origin of host species (Fig. 1). For example, all arthropod LARP4 proteins are PAM2 deficient, contrary to a decapod and a cnidarian LARP4. Even more surprising is the finding of PAM2containing LARP4 proteins in gastropod, echinoderm, and cephalochordate species, while LARP4 from a urochordate species lacks this motif (Fig. 1). Curiously, the PAM2-like of invertebrate LARP4 (when present) is more canonical (Kozlov et al. 2010) than the vertebrate ones in that it contains the consensus phenylalanine residue instead of the tryptophan (Fig. 2A). The transition from phenylalanine to tryptophan in the PAM2-like sequence can be observed in cephalocordates and hence predates the radiation of the vertebrate lineage and the gene duplication event specific to that lineage. It is therefore likely that the acquisition of PAM2 predates the radiation of the vertebrates; however, this motif was then either fixed or lost in the different invertebrate lineages, suggesting that it was not under strong selective pressure. The situation apparently changed in the vertebrate common ancestor, where a functional, although atypical, PAM2 was fixed and remained conserved in all vertebrate lineages. A summary of the domain organization of eukaryote LARP4 proteins is presented in Supplemental Figure S1A.

The LAM is extremely conserved at the primary sequence level, across every LA-motif-containing protein. In the LAM consensus, positions Q20, Y23, Y24, D33, F35, and F55 were found previously to be particularly well conserved (Bousquet-Antonelli and Deragon 2009; Bayfield et al. 2010). Functional and structural studies conducted on the genuine La proteins unambiguously delineated the role of these amino acids in mediating the $3^{\prime} \mathrm{UUU}-\mathrm{OH}$ RNA recognition in the context of the La module (Dong et al. 2004; Teplova et al. 2006; Kotik-Kogan et al. 2008; Bayfield et al. 2010; Martino et al. 2012).

By aligning the LAM from protist and invertebrate LARP4, a very good conservation of these key amino acids 
A.

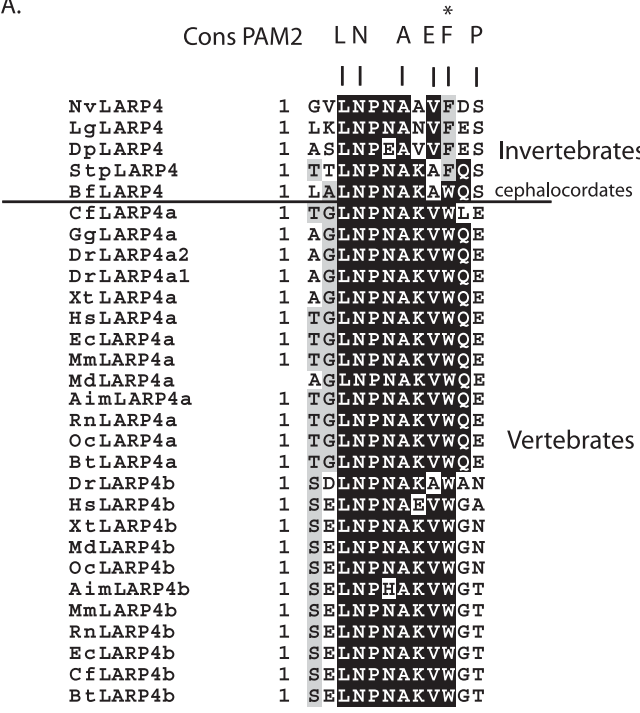

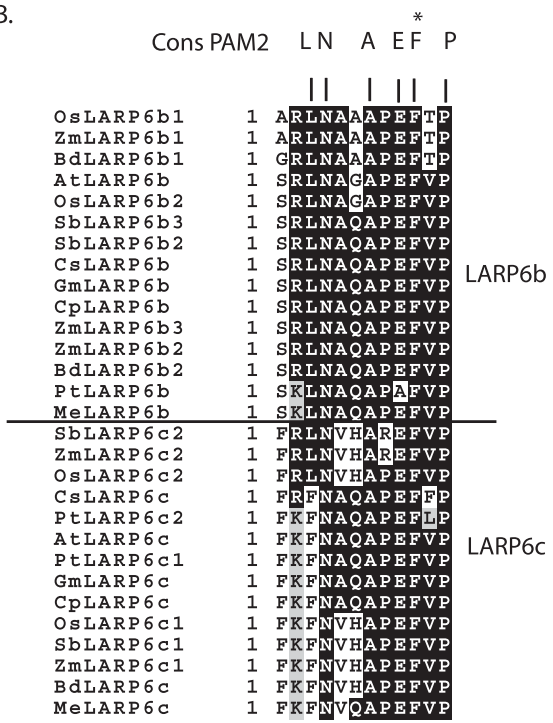

FIGURE 2. Alignment of the putative PAM2 present in LARP4 $(A)$ and LARP6 (B) proteins. The most conserved positions of the eukaryote PAM2 are indicated above each alignment. $\left(^{*}\right)$ The position of an invariant phenylalanine proposed to be essential for binding to the PABP MLLE motif (Kozlov et al. 2010). A dark line separates PAM2 from invertebrate and vertebrate LARP4. The conserved phenylalanine was replaced by a tryptophan starting from cephalocordates, thus before the gene duplication events that happened early in the vertebrate lineage. For LARP6, PAM2s are only found for vascular plant proteins that belong to LARP6b or LARP6c subgroups.

was observed (Fig. 1; Supplemental Fig. S2), contrary to the vertebrate LARP4 proteins, where several possibly nonconservative substitutions could be seen, suggesting a reorganization of the LAM in that lineage (Fig. 1; Supplemental Fig. S2). Since the LAM is likely to work in association with the flanking RRM-like domain to bind RNA, this was also subjected to analysis. Although a number of amino acid changes distinguish vertebrate LARP4a from LARP4b, the predicted structural arrangement of the RRM-like region appears similar in protist, invertebrate, and vertebrate LARP4 (Supplemental Fig. S3), apparently confining the reorganization to the LAM. Taken together, these observations suggest that in the vertebrate lineage a gene duplication event associated with the presence of a functional PAM2 is at the basis of an important reorganization of the LAM and possibly of LARP4 RNA-binding properties.

\section{Eukaryote LARP6 evolutionary history}

The LARP6 family has a broader distribution than the LARP4 family being represented not only in protists and animals but also in the "green" lineage (green algae, mosses, and vascular plants) (Bousquet-Antonelli and Deragon 2009). To explore in more detail the evolutionary history of the LARP6 family in eukaryotes, we performed a phylogenetic analysis using 41 LARP6 proteins from 28 protist, plant, and animal species (Supplemental Table S1). In protists, LARP6 is encoded by a single gene, found thus far only in genomes of oomycetes species (Fig. 3), and the same occurs in invertebrates. Conversely, with the exception of eutherians, vertebrate LARP6 is encoded by two genes, the products of which are named LARP6a and LARP6b (Fig. 3). Akin to the LARP4 family, this situation results likely from the duplication of the LARP6 gene in the common ancestor of the vertebrate lineage $(\sim 525$ million yr ago, Mya). However, in the case of LARP6, this gene duplication was followed much later (125 Mya) by the loss of one of the two copies (LARP6b) in the common ancestor of eutherians. A comparison of the La module of LARP6/LARP6a with LARP6b reveals no significant differences (data not shown); however, it emerged that eutherian LARP6 and vertebrate LARP6a, but not LARP6b, contain a conserved nuclear export signal (NES) and nuclear localization signal (NLS) (see Supplemental Fig. S4) that were shown previously to be functional in the context of the human LARP6 (Valavanis et al. 2007). These observations suggest that LARP6b may have the same mRNA targets as LARP6a/LARP6 (i.e., $\alpha 1 / \alpha 2$ chains of collagen I and III) but different subcellular trafficking (see Discussion).

Next, we examined the evolutionary history of LARP6 in the green lineage (Fig. 4) and found that while in green algae and mosses a single gene encodes for LARP6, the number of LARP6 genes ranges from three to six in vascular plants, with most of the dicotyledon species having three genes and most of the monocotyledon species having six. The phylogeny of these proteins clearly clusters them in three distinct groups (named a, b, and c) (Fig. 4). Notably, whereas plant LARP6a is always encoded by a single gene in all species examined, several genes may encode for different LARP6b and LARP6c versions. Our analysis also revealed that plant LARP6b and LARP6c always comprise a putative PAM2 in their N-terminal region (see Figs. 2B, 4), but this 


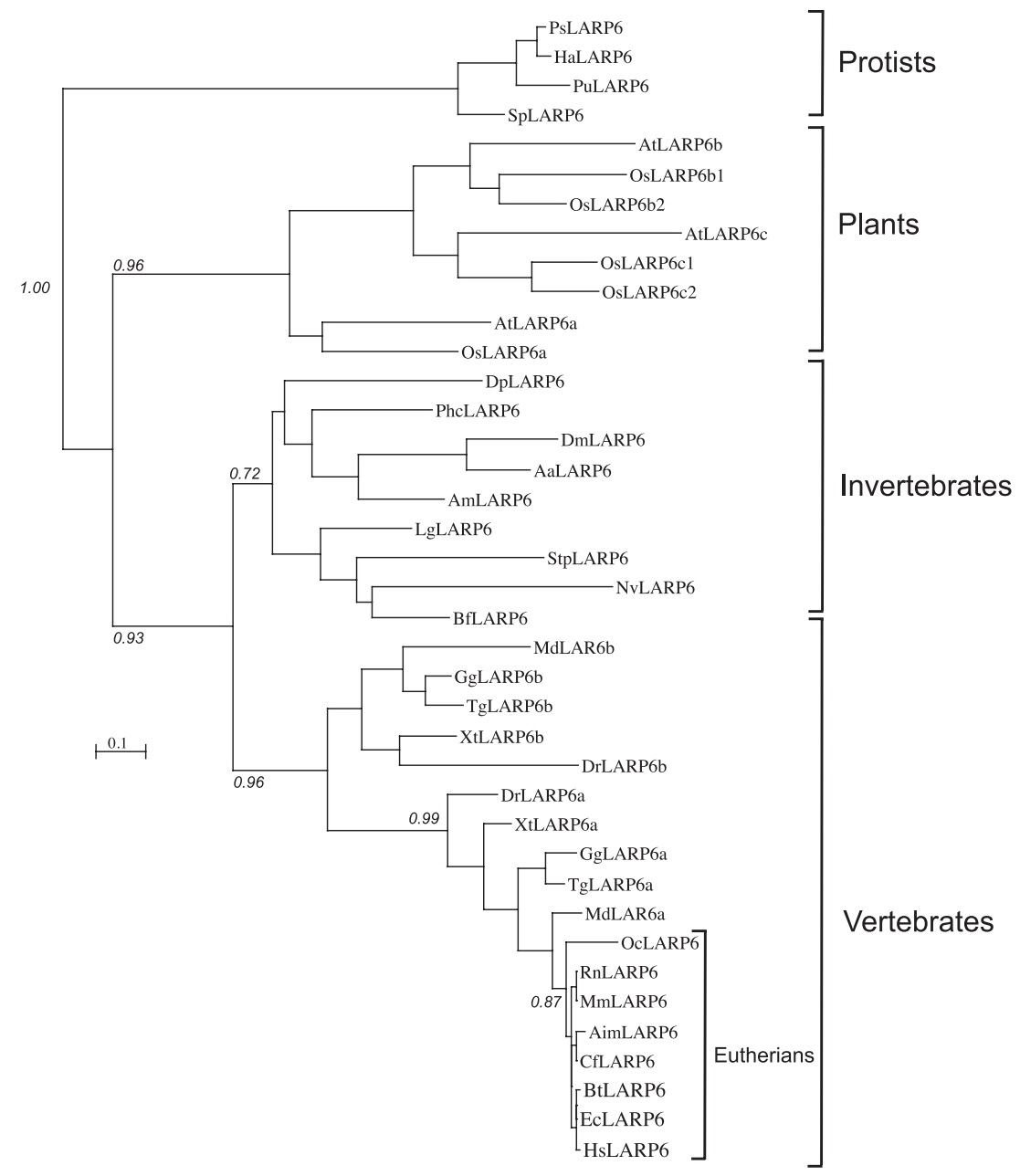

FIGURE 3. Detailed phylogenetic analysis of the LARP6 family. The phylogenetic tree was obtained using La module (LAM-RRM) sequences of 39 LARP6 proteins form protist, plant, invertebrate, and vertebrate species (see Supplemental Table S1 for protein sequences). Selected informative statistical supports (approximate likelihood-ratio test [aLRT] data) are indicated. Species codes are as in Figure 1 with the following additions: (Ha) Hyaloperonospora arabidopsidis; (Pu) Pythium ultimum; (Sp) Saprolegnia parasitica; (At) Arabidopsis thaliana; (Os) Oryza sativa.

motif is consistently missing in LARP6a. A summary of the domain organization of eukaryote LARP6 proteins is presented in Supplemental Figure S1B.

The plant LARP6b PAM2 sequence is closer to the eukaryote consensus PAM2 than the one found in vertebrate LARP4, because all consensus amino acids are conserved (Fig. 2). This is not the case for most plant LARP6c PAM2s, which possess a phenylalanine instead of the consensus leucine in position 3 (Fig. 2B). This position as been shown to be very important for binding and very sensitive to mutation (Lim et al. 2006; Kozlov et al. 2010). However, in two cases (for the human NF-X1 and TOB2 proteins), a PAM2 also presenting a leucine-to-phenylalanine substitution at the same position was found to be involved in binding to PABP (Okochi et al. 2005; Lim et al. 2006; Katzenellenbogen et al. 2007; Kozlov et al. 2010).
Strikingly, analogously to the LARP4 family, the acquisition of this putative PAM2 correlates with a significant reorganization of the LARP6 LAM (Fig. 4). In particular, PAM2-lacking LARP6 proteins, such as algae and moss LARP6 and vascular plant LARP6a (Fig. 4; Supplemental Fig. S5), show a very good conservation of the key LAM amino acids previously shown in La to be involved in RNA binding. Conversely, and akin to vertebrate LARP4, the acquisition of a putative PAM2 in the common ancestor of the $6 \mathrm{~b}$ and $6 \mathrm{c}$ lineages was found consistently coupled with what appears to be nonconservative amino acid replacements of these LAM residues (see Fig. 4; Supplemental Fig. S5).

To further test the importance of these six LAM residues and their overall level of conservation in eukaryotes, we aligned the LAM of 234 proteins from 78 species of protists (18 species), yeasts (24 species), animals (23 species), and plants and algae (13 species) belonging to the different LARP1, 4, 6, 7, and genuine La families (Supplemental Fig. S6). We deliberately excluded from this analysis LAMs from vertebrate LARP4a and $b$ and plant LARP6b and $c$ that are all associated with a PAM2 motif. We observed an extremely high level of conservation of these six positions for LAMs not associated with a PAM2 motif. Position 20 of these LAMs is occupied by a $\mathrm{Q}$ in $96.9 \%$ of the protein tested; position 23 by an $\mathrm{F}, \mathrm{Y}$, or $\mathrm{W}$ in $97.9 \%$ of the cases; position 24 by an F, Y, or W in $99.7 \%$ of the cases; position 33 by a $\mathrm{D}$ in $99.7 \%$ of the cases; position 35 by an $\mathrm{F}$ or $\mathrm{Y}$ in $96.2 \%$ of the cases; and position 55 by an $\mathrm{F}$ in $97.6 \%$ of the cases (Supplemental Fig. S6). Such high level of sequence conservation over $>2$ billion yr of evolution is impressive but could still be underestimated since some proteins included in this analysis may be nonfunctional pseudogenes. From this analysis, we conclude that deviations from the observed consensus for these six positions only arise in LAMs associated to PAM2 motifs and are likely to deeply affect its biophysical properties.

Moreover, we observed that, in the case of plant PAM2containing LARP6b and LARP6c, and contrary to what is observed for the vertebrate LARP4 (Supplemental Fig. S3), the RRM-like region also underwent modifications compared with LARP6 versions without PAM2 (see Supplemental Fig. S7). Indeed, the size of the loop3 region (between 


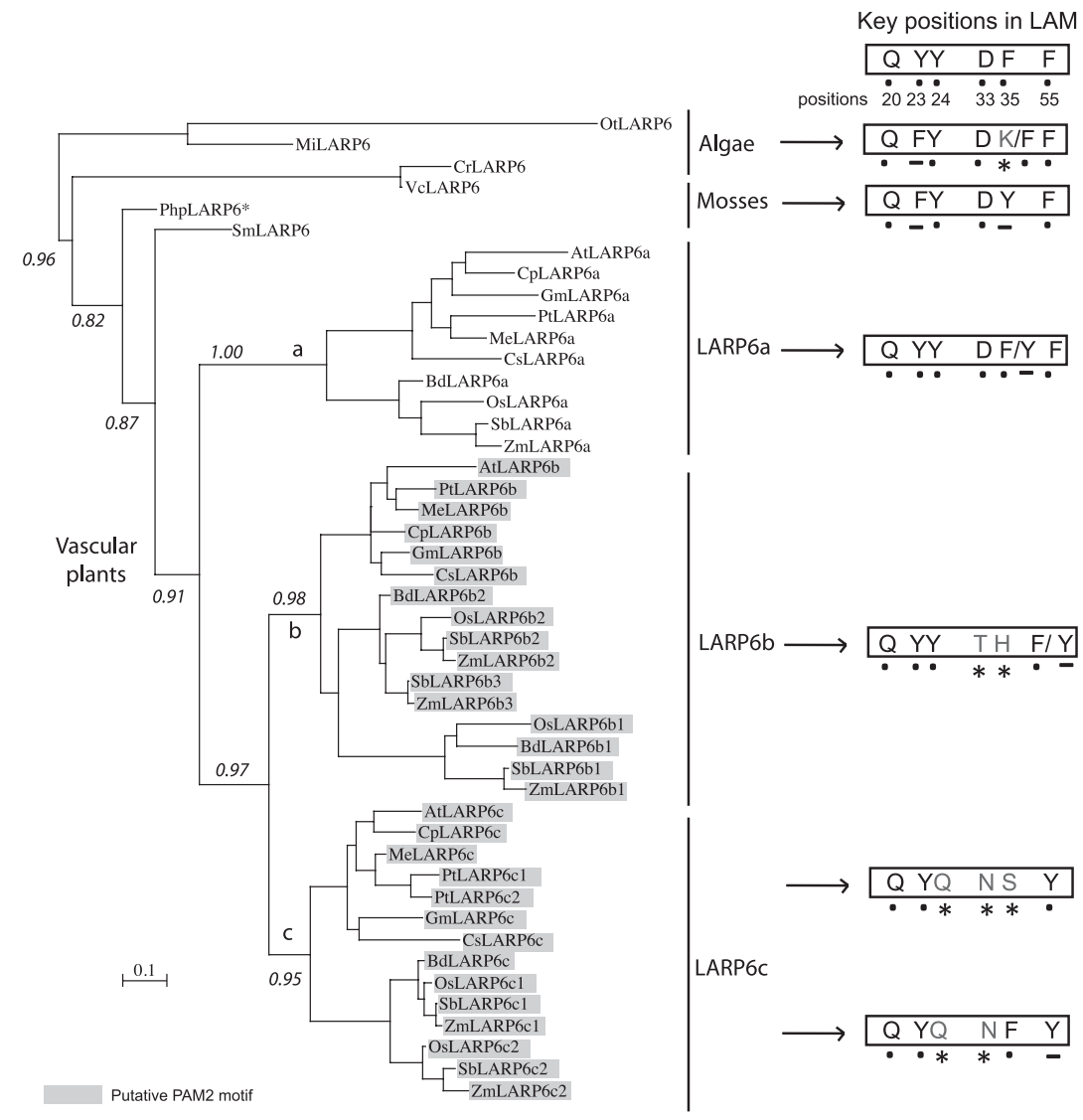

FIGURE 4. Phylogenetic analysis of the LARP6 family in the green lineage. The phylogenetic tree was obtained using La module (LAM-RRM) sequences of 46 LARP6 proteins from green algae, mosses, and vascular plant species (see Supplemental Table S1 for protein sequences). Selected informative statistical supports (approximate likelihood-ratio test [aLRT] data) are indicated. (Gray) Proteins presenting a putative PAM2 (see Fig. 2B). Key positions of the LAM, known to form the RNA-binding pocket in the context of the genuine La protein (Bayfield et al. 2010), are indicated on the right (dots indicate conserved amino acids, underlined amino acids are for possible conservative changes, and gray amino acids with an asterisk are for possible nonconservative changes). The numbering refers to the position of the amino acids in the genuine human LAM. Species codes are (Ot) Ostreococcus tauri; (Mi) Micromonas; (Vc) Volvox carteri; (Cr) Chlamydomonas reinhardii; (Php) Physcomitrella patens; (Sm) Selaginella moellendorfii; (At) Arabidopsis thaliana; (Cp) Carica papaya; $(\mathrm{Gm})$ Glycine max; (Pt) Populus trichocarpa; (Me) Manihot esculenta; (Cs) Cucumis sativis; (Bd) Brachypodium distachyon; (Os) Oryza sativa; (Sb) Sorghum bicolor; (Zm) Zea mays.

the $\beta$-strands $\beta 2$ and $\beta 3$ ) is predicted to be invariant for all plant LARP6as but is larger for LARP6b and much more variable for the LARP6b and LARP6c proteins (Supplemental Fig. S7). Loop3 is a critical region usually responsible for direct contacts with RNA substrates in many canonical and noncanonical RRM proteins (Maris et al. 2005; Clery et al. 2008) and was also shown to be important for pre-tRNA interaction in the RRM1 of genuine La (Bayfield and Maraia 2009). In contrast, the very long (45-amino-acid) loop3 region of the noncanonical RRM2 present in the Tetrahymena LARP7 protein was found not to be involved in RNA binding (Singh et al. 2012). Therefore, it remains to be seen if the observed structural reorganization of the LARP6b and LARP6c RRM-like domain following PAM2 acquisition contributes to modifying the RNA-binding properties of these proteins.

\section{PABP-binding properties of plant PAM2-containing LARP6 proteins}

To characterize experimentally the putative PAM2 motifs of plant LARP6 proteins uncovered by our bioinformatics investigations, we examined their ability to interact in vitro with the MLLE domain (comprising residues 539-642 as defined in Siddiqui et al. 2007) of the major Arabidopsis thaliana poly(A)binding protein (AtPAB2) (Palanivelu et al. 2000a,b). The interaction of recombinant AtPAB2 MLLE with peptides spanning, respectively, the putative PAM2 of AtLARP6b (PAM6b) and AtLARP6c (PAM6c) was analyzed using Isothermal Titration Calorimetry (ITC) (Fig. 5). The ITC experiments confirmed that both peptides engage in a 1:1 complex with AtPAB2 MLLE. The binding affinities (dissociation constant, $K_{\mathrm{d}} \sim$ 1.5-2 $\mu \mathrm{M}$ ) and the thermodynamic signature for both interactions (Supplemental Table S2) are very alike and also adhere closely to the interaction profile of the wheat PABP MLLE with a canonical PAM2 from a wheat protein previously reported by Siddiqui et al. (2007). These results, together with the primary sequence conservation of LARP6 PAM2 (Fig. 2B), support the hypothesis that a phenylalanine can substitute for a leucine in position 3 of the motif without affecting binding in vitro.

To determine whether LARP6a, 6b, and $6 \mathrm{c}$ are able to form a complex in vivo with the PABP, the three proteins were, respectively, transiently coexpressed (as GFP-fused proteins) with AtPAB2 (fused to tagRFP) in Nicotiana benthamiana leaves and coimmunoprecipitation experiments performed with anti-GFP antibodies (Fig. 6). In agreement with our hypothesis, AtPAB2 was present in the GFP-LARP6b and GFP-LARP6c immunoprecipitates (Fig. 6, lanes 4,8), but was not detected in the GFP-LARP6a bound fraction (Fig. 6 , lane 2). To test for the presence of the tagRFP-PAB2 protein, the input and eluate fractions were analyzed by Western blot with an anti-PAB2 antibody that was raised against the full-length Arabidopsis protein (Dufresne et al. 2008) and displays the ability to recognize the $N$. benthamiana PABPs (data not shown) (see the input lanes 

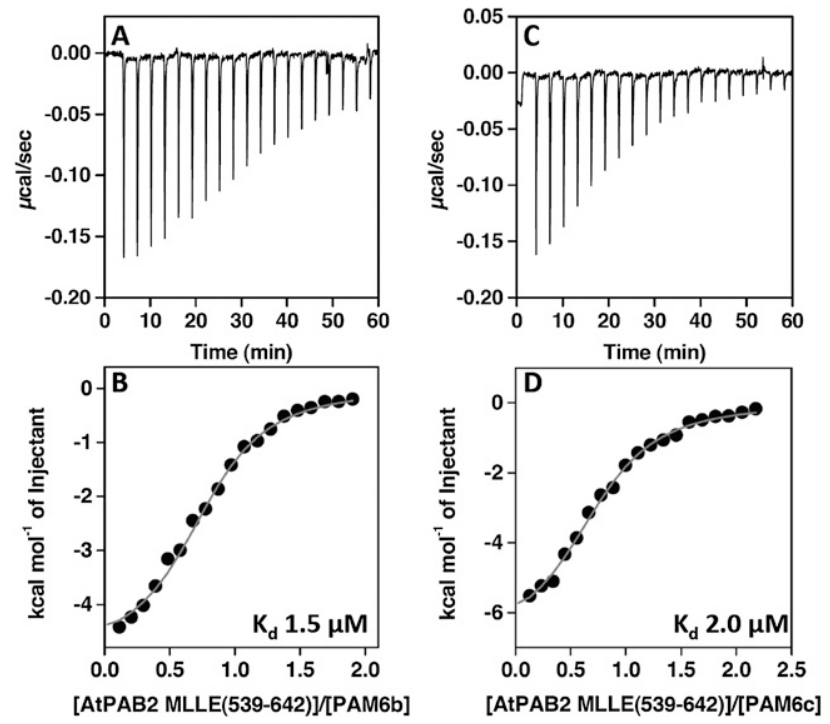

FIGURE 5. Calorimetric analysis of the interaction between the AtPAB2 MLLE domain (539-642) and the peptides PAM6b and PAM6c. Raw titration data showing the thermal effect of injecting an AtPAB2 MLLE solution into a calorimetric cell containing peptide PAM6b $(A)$ or peptide PAM6c $(C)$. The normalized heat for the titrations, shown in $B$ and $D$, respectively, was obtained by integrating the raw data and subtracting the heat of the protein dilution. The gray lines in $B$ and $D$ represent the best fit derived by a nonlinear least-squares procedure based on an independent binding sites model. The dissociation constant $K_{\mathrm{d}}$ is indicated for each interaction, and the thermodynamic parameters are shown in Supplemental Table S2.

from Fig. 6). We observe that we detect not only the Arabidopsis PAB2 protein in the eluate fraction of GFP-6b and $6 \mathrm{c}$ experiments but also the NbPABP, further sustaining the PABP-interacting properties of AtLARP6b and $6 \mathrm{c}$ factors.

Several proteins such as Paip1 and Paip2 bind the PABP not only with their PAM2 motifs but also with additional domains (Khaleghpour et al. 2001a; Roy et al. 2002). To prove that LARP6b and 6c PAM2 motifs are, indeed, functional in vivo and determine their contribution to PABP binding, we repeated the previously described coimmunoprecipitation assays with GFP-tagged $6 \mathrm{~b}$ and $6 \mathrm{c}$ deleted of their PAM2 regions (corresponding to the PAM2-6b and PAM2-6c peptides used for the ITC experiments shown in Fig. 5). In each eluate fraction, we quantified the tagRFP-PAB2 and NbPABPs signals and normalized them to their respective amount of immunoprecipitated GFP signal. We then compared the mutant ratios to the full-length ratios and found that deletion of the PAM2 domains impairs immunoprecipitation of the Arabidopsis and Nicotiana PABPs (Fig. 6, cf. lanes 4 and 6, lanes 8 and 10). The LARP6b-PABP complex formation is almost completely abolished with only $7 \%$ and $<1 \%$ PABP proteins detected in the mutant eluate fraction (Fig. 6, lane 6), while the LARP6c-PABP complex formation is more modestly affected with $75 \%$ and $35 \%$ of PABPs remaining in the eluate fraction (Fig. 6, lane 10).

In summary, these experiments suggest that LARP6b and $6 \mathrm{c}$ are PABP-interacting factors in planta, while LARP6a does not display the ability to bind to the poly(A)-binding protein. The PAM2-6b and PAM2-6c domains are not only functional in vitro (Fig. 5) but also in vivo with most if not all of the LARP6b-PABP interaction endorsed by its PAM2 motif. The LARP6c binding to PABP likely also relies on an MLLE-PAM2 interaction, but the involvement of its PAM2 is not as strong as for the PAM2-6b. We propose that LARP6c relies on additional domain(s) to bind to PABP, while LARP6b does not, or to a much lesser extent. Finally, these results suggest that while the leucine-to-phenylalanine divergence at position 3 of the PAM2-6c domain does not impair its ability to interact with a canonical PABP-MLLE in vitro, it might have an impact in the context of the full-length protein in vivo.
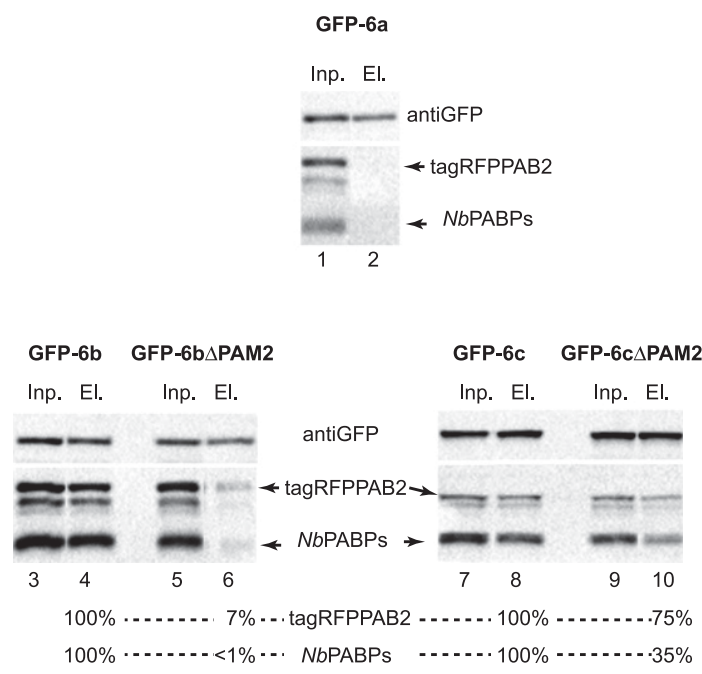

FIGURE 6. Plant PAM2-containing AtLARP6b and AtLARP6c can form a complex with AtPAB2. GFP-tagged versions of AtLARP6a, $6 \mathrm{~b}, 6 \mathrm{c}, 6 \mathrm{~b} \triangle \mathrm{PAM} 2$, or $6 \mathrm{c} \Delta \mathrm{PAM} 2$ were, respectively, coexpressed in $N$. benthamiana leaves with AtPAB2 fused to the tagRFP label (a fluorescent tag from E. quadricolor that is not recognized by antiGFP antibodies) (Merzlyak et al. 2007). Immunoprecipitations were performed using the anti-GFP antibodies. Western blot analyses using either anti-GFP or anti-PAB2 antibodies on the input (Inp; $3.5 \%$ of the total input) or eluate (El; $50 \%$ of the eluate) fractions are shown. The positions of the GFP fusions (GFP), tagRFPPAB2, and $N$. benthamiana PABP (NbPABPs) are indicated. The intensities of the GFP, tagRFPPAB2, and NbPABPs signals in eluate fractions of the $6 \mathrm{~b}, 6 \mathrm{~b} \Delta \mathrm{PAM} 2$ and $6 \mathrm{c}, 6 \mathrm{c} \Delta \mathrm{PAM} 2$ have been quantified and compared. Each eluate signal (GFP, tagRFPPAB2, NbPABPs) has been quantified and divided by its corresponding value in the input. Then the tagRFP and NbPABP obtained values have been divided by the GFP signal from the same eluate. This gives normalization to the pull-down efficiency. We arbitrarily set the eluate values obtained for the pull-down experiments with the full-length proteins to $100 \%$ and give the values of the remaining signals in the eluates fractions of the $\triangle$ PAM2 pull-downs. 


\section{RNA-binding properties of plant LARP6 proteins}

To test the hypothesis that the observed reorganization of AtLARP6 RNA-binding motifs upon PAM2 acquisition (Fig. 4; Supplemental Figs. S5, S7) resulted in a significant change of RNA-binding properties for the proteins, we set out to measure by ITC the binding affinity of the La module of AtLARP6a, b, and c for several homopolymers (Figs. 7, 8; Supplemental Figs. S8, S9). Unfortunately, although the protein was successfully expressed, the analysis of the AtLARP6b RNA-binding profile was prevented by the inability to obtain well-behaved, stable and/or monodisperse protein for rigorous and reproducible ITC analysis, despite the numerous attempts of varying domain boundaries, tags and purification protocols of the recombinant protein, as well as experimental conditions of the ITC measurements (data not shown). Data presented here therefore concern AtLARP6a and AtLARP6c, for which protein fold and stability were checked by ${ }^{1} \mathrm{H}$ NMR (data not shown).

AtLARP6a bound to all homopolymers tested, albeit exhibiting higher affinity for 20-nt oligo(A) (Fig. 7; Supplemental Fig. S8; Supplemental Table S2) (20-nt oligo(A)> $\mathrm{U}>\mathrm{C}, \mathrm{G})$. Given that ITC also provides the stoichiometry and thermodynamics description of the interaction, we were able to observe that in all cases the binding is centered on a molar ratio of 1 and is enthalpically driven (Supplemental Table S2). Furthermore, experiments conducted with homopolymeric 10-nt oligo(A) and oligo(U) revealed that the binding is somewhat length dependent, since the binding affinity decreased with the shorter oligonucleotides (Supplemental Fig. S8; Supplemental Table S2). Genuine La proteins from many species recognize the $3^{\prime} \mathrm{OH}$ group, a specificity provided by the aspartic acid in position 33 of the LAM (according to the amino acid numbering of the human protein) (Teplova et al. 2006; Kotik-Kogan et al. 2008), and are therefore very sensitive to the introduction of a 3'O-phosphate on the RNA ligand. Since D33 of human La protein LAM is conserved in AtLARP6a, two such variants [20-nt oligo(A)-3'O-phosphate and 20-nt oligo(U)-3'O-phosphate] were analyzed for their ability to interact with this protein. Supplemental Figure S8 shows that this modification had no observable impact on the affinity of AtLARP6a for either of the RNA tested, suggesting that the binding mechanism for this protein is different from that of genuine $\mathrm{La}$ in that it does not involve the $3^{\prime} \mathrm{OH}$ recognition. The RNA-binding properties of AtLARP6a are therefore similar to those reported for HsLARP4a (Yang et al. 2011), both proteins having a preference for poly(A) over poly(U) (albeit more so for HsLARP4a), a length dependency and a lack of 3' termini recognition despite the conservation of the key aspartic acid residue.

Contrary to AtLARP6a and to HsLARP4a, AtLARP6c showed a strong preference for single-stranded 20-nt oligo(U), exhibiting $\sim 10$-fold weaker affinity for 20 -nt oligo(A) and oligo(G) and no observable binding, i.e., beyond the threshold that could be rigorously measured by ITC, for 20-nt oligo(C) (Fig. 8). Similarly to AtLARP6a and HsLARP4a, but exhibiting even a more pronounced effect, RNA recognition of AtLARP6c appears to depend on the length of the homopolymer used (Supplemental Fig. S9). Equally, it is independent from $3^{\prime}$ termini recognition since the 3'O-phosphorylated 20-nt oligo(U) RNA gave a binding profile analogous of the $3^{\prime} \mathrm{OH}$ counterpart (Supplemental Fig. S9; Supplemental Table S2).
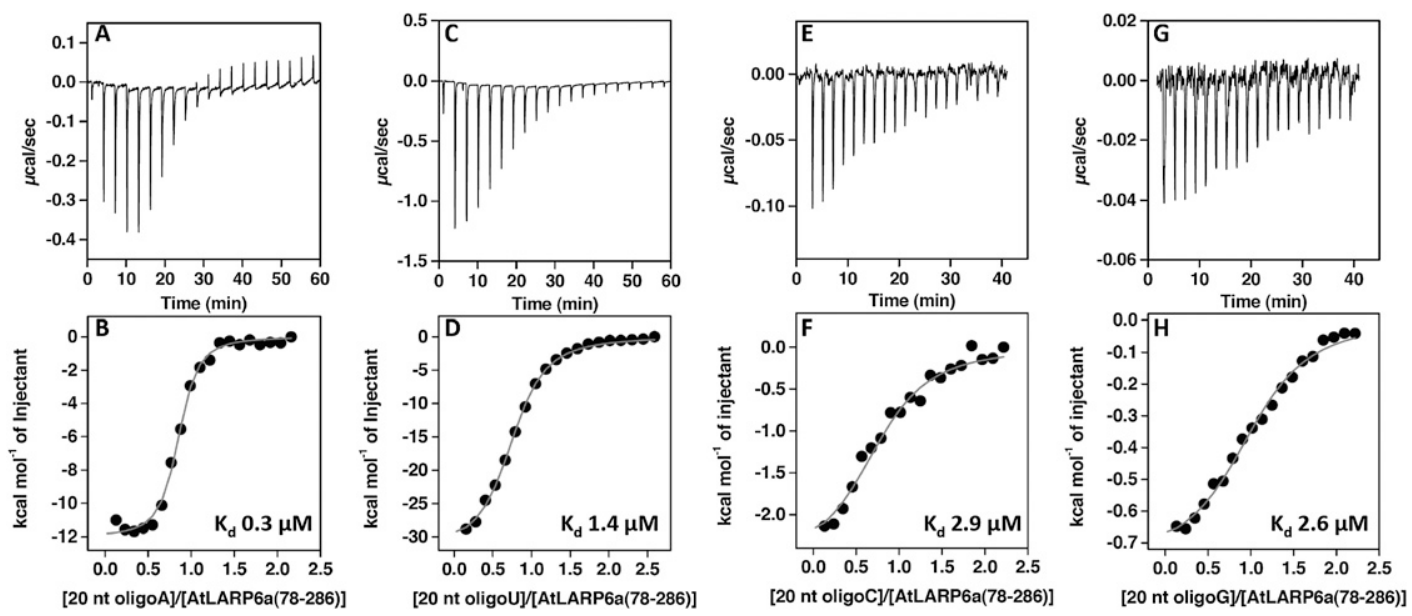

FIGURE 7. RNA-binding properties of AtLARP6a. ITC experiments were conducted with four RNA targets: 20-nt oligo(A) (A,B), 20-nt oligo(U) $(C, D)$, 20-nt oligo $(C)(E, F)$, and 20-nt oligo $(G)(G, H)$. Integration of the raw titration data $(A, C, E, G)$ generated sigmoid-shaped curves $(B, D, F, H$, respectively) centered on a 1:1 molar ratio that are interpolated by an equivalent and independent binding model (gray lines represent the best fit). The dissociation constant $K_{\mathrm{d}}$ is indicated for each RNA target, and the thermodynamic parameters are shown in Supplemental Table S2. All the RNA sequences tested bind to AtLARP6a, but 20-nt oligo(A) interacts approximately fivefold stronger than 20-nt oligo(U) and ninefold stronger than 20-nt oligo(C) and 20-nt oligo(G). 

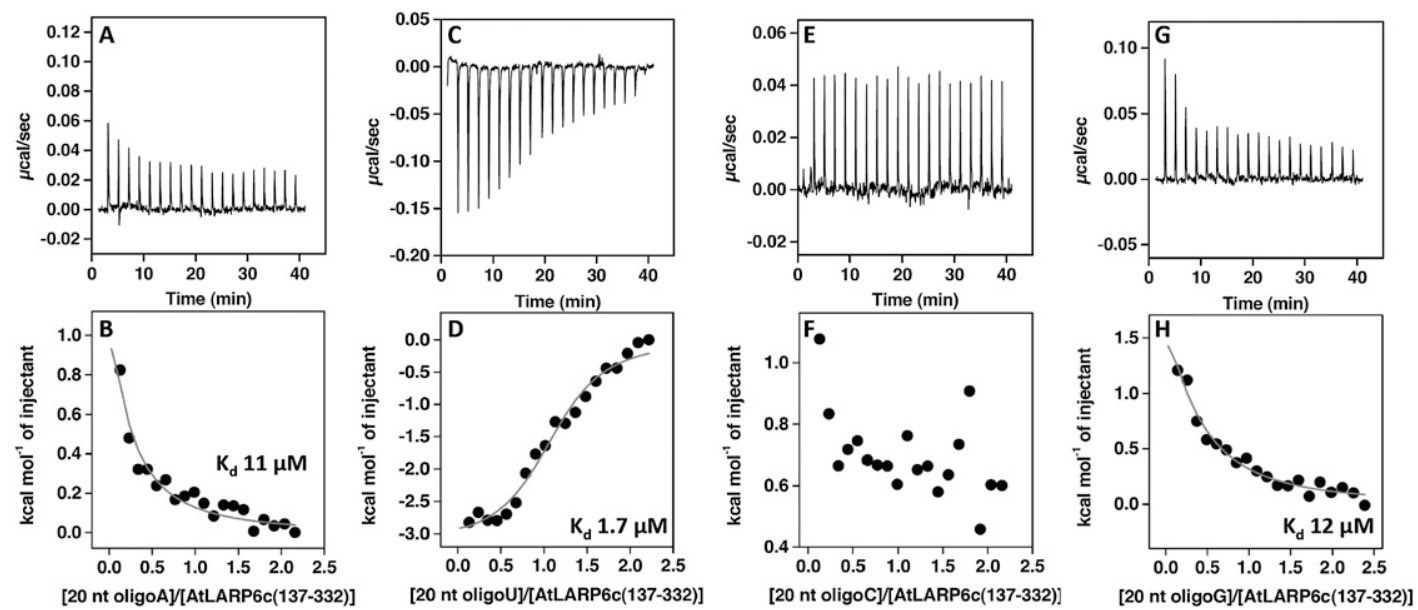

FIGURE 8. RNA-binding properties of AtLARP6c. ITC experiments were performed with four RNA targets: 20-nt oligo(A) $(A, B)$, 20-nt oligo(U) $(C, D)$, 20-nt oligo $(C)(E, F)$, and 20-nt oligo $(G)(G, H)$. Integration of the raw titration data $(A, C, G)$ generated sigmoid-shaped curves $(B, D, H$, respectively) centered on a 1:1 molar ratio that are interpolated by an equivalent and independent binding model (gray lines represent the best fit). Nonetheless, the fit is poorer for 20-nt oligo(A) and 20-nt oligo $(\mathrm{G})(B, H$, respectively) because of the weaker interaction. No interaction could be detected by ITC with 20-nt oligo $(C)(F)$. The dissociation constant $K_{\mathrm{d}}$ is indicated, and the thermodynamic parameters are shown in Supplemental Table S2. AtLARP6c shows a clear preference for 20-nt oligo(U), binding to 20-nt oligo(A), and 20-nt oligo(G) with a sevenfold lower affinity.

Although these experiments constitute an initial screening of the RNA-binding profile of these proteins, using homopolymeric single-stranded RNA sequences and their variants, they suggest that the RNA-binding properties of AtLARP6a and AtLARP6c are significantly different (see Discussion).

\section{Subcellular localization of plant LARP6 proteins}

To further test the hypothesis that PAM2 acquisition was associated to the neofunctionalization of plant LARP6, we determined the subcellular localization of tagged versions of AtLARP6a, b, and c following their transient expression in onion epidermal cells (Fig. 9). The proteins were coexpressed with a nucleolar marker (the Arabidopsis homolog of the NOP10 nucleolar protein) or the AtPAB2 as marker of the stress granules. We found that as expected, the GFPNOP10 protein is highly enriched in the nucleolus and does not form aggregates under stress conditions (Fig. 9A, panels b,d; Fig. 9B, panels b,d and e-g; Fig. 9C, panels a-c, for stressed conditions). The AtPAB2 under normal conditions displays a cytoplasmic diffuse pattern and is excluded from the nuclear compartment (data not shown). We found that AtLARP6a is highly enriched in the nucleolus, as assessed by colocalization with AtNOP10 (Fig. 9A, panels a-d), present in the cytoplasm (Fig. 9A, panels a,e) but mainly absent from the nucleoplasm (Fig. 9A, panels a,d). Furthermore, cytoplasmic AtLARP6a does not aggregate upon hypoxic stress (Fig. 9A, panels e-g). The PAM2-containing AtLARP6b displayed the same subcellular localization observed for AtLARP6a (Fig. 9B, panels a-d); however, contrary to the latter, upon hypoxia AtLARP6b forms aggregates (Fig. 9B, panels e-g) that colocalize with
AtPAB2 to subcytoplasmic foci (Fig. 9B, panels i-k) that are most likely stress granules, AtPAB2 being a classical marker of such mRNP granules (Weber et al. 2008). The other PAM2-containing protein, AtLARP6c, is present both in the nucleoplasm and nucleolus (Fig. 9C, panels a-c) as well as in the cytoplasm (Fig. 9C, panels e,f). Nevertheless, akin to AtLARP6b and in contrast with AtLARP6a, AtLARP6c also forms subcytoplasmic aggregates (Fig. 9C, panel a) that colocalize upon stress with AtPAB2 in stress granules (Fig. 9C, panels $\mathrm{g}-\mathrm{i}$ ).

Since stress granules are proposed to be involved in mRNA storage, recycling, or degradation (Anderson and Kedersha 2009), the subcellular localization of AtLARP6b and AtLARP6c suggests that these proteins acquired a function in mRNA metabolism, at least in stress conditions (see Discussion).

\section{DISCUSSION}

\section{Evolution of LARP4 and LARP6 families}

In this study, we show that at two independent times in evolution (during vertebrate evolution for LARP4 and vascular plant evolution for LARP6), the duplication of LARP genes was followed by the acquisition of a PAM2 and by an evident reorganization of the LAM RNA-binding pocket (Figs. 1, 4; Supplemental Fig. S6). Since in genuine La significantly different RNA-binding properties can be achieved without altering the LAM per se (Martino et al. 2012), such reorganization was somewhat unexpected. Duplication of LARP genes is not as a rule accompanied by these modifications; for example, vascular plant LARP6a 
A
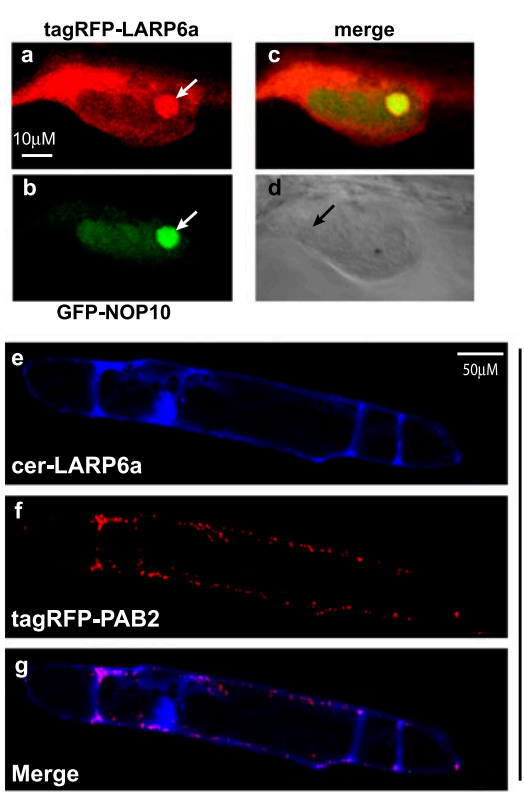

C

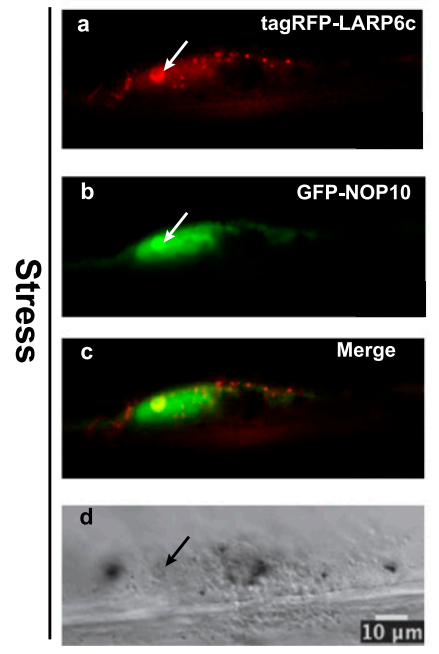

B
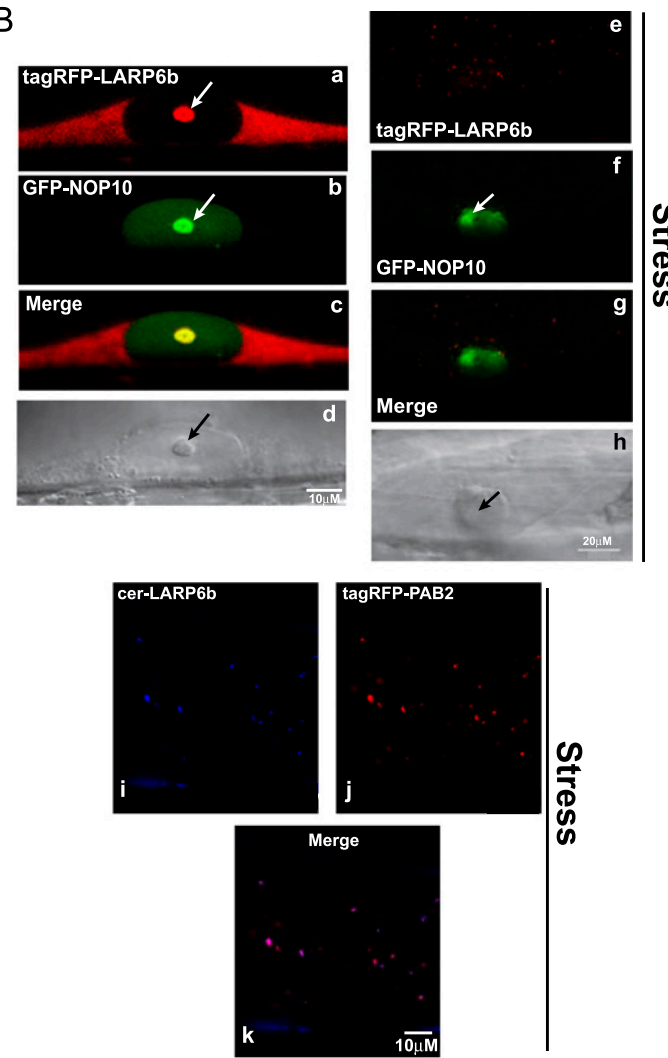
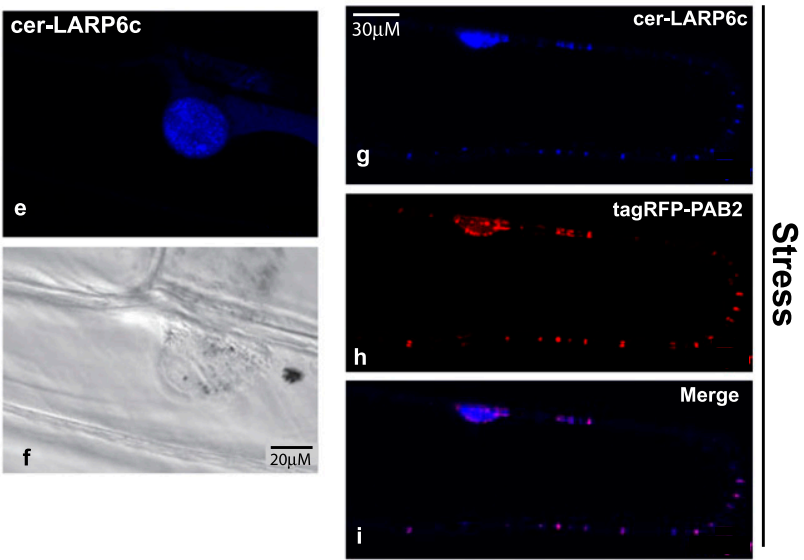

FIGURE 9. Subcellular localization of plant LARP6. TagRFP-labeled AtLARP6a $(A), 6 \mathrm{~b}(B)$, or $6 \mathrm{c}(C)$ proteins were transiently expressed in onion epidermal cells, and their subcellular localization was analyzed by confocal microscopy. tagRFP-tagged versions of the AtLARP6a, 6b, or 6c were, respectively, coexpressed with GFP-NOP10 and Cerulean (cer)-tagged versions of AtLARP6a, 6b, or 6c were, respectively, coexpressed with a tagRFP-labeled AtPAB2 protein in onion epidermal cells, and the subcellular localization of each fusion protein was analyzed and compared in stress situations (hypoxia). Arrows point to the nucleoli.

as well as vertebrate LARP6a and LARP6b still possess conventional LAM in the context of multiple versions of the protein (Figs. 3, 4), leading to the suggestion that PAM2 acquisition is, indeed, causal in this reorganization. This hypothesis is supported by the systematic presence of a conventional LAM for all protist, plant, and animal LARPs lacking a PAM2 motif and a mutated LAM for most protist, plant, and animal PAM2-containing LARPs (Figs. 1, 4).
Given that the PABP-binding capacity conferred by PAM2 (Figs. 5, 6; Yang et al. 2011) allows the direct targeting of PAM2-containing LARPs to mRNA, we propose this to be a major driving force responsible for the restructuring of their LAM and for their final neofunctionalization. This evolutionary scenario implies that plant LARP6as that do not possess PAM2 and have a "classical" LAM pocket are likely to fulfill a distinct "ancestral" function compared with 
LARP6b and c subfamilies' members. Similarly, protist and invertebrate LARP4 and LARP6, characterized by the absence of a PAM2 motif and by a "classical" LAM pocket (Figs. 1, 3), plausibly execute a yet-undefined "ancestral" function compared with their vertebrate orthologs.

The situation of vertebrate LARP6 is interesting seeing that this protein probably evolved in the vertebrate lineage to accommodate new substrates, i.e., the collagen $5^{\prime}$-UTR mRNAs (Cai et al. 2010a,b). A gene duplication event in the common ancestor of vertebrates is likely to be at the origin of this evolution, but in this case without the occurrence of PAM2 acquisition or LAM significant modifications. This is consistent with the fact that the vertebrate LARP6 La module binds a specific stem-loop structure in the $5^{\prime}$-UTR region of only a few mRNAs (Cai et al. 2010a; MR Conte, unpubl.), without the need to interact with PABP for its highly specialized function. Surprisingly, this gene duplication event was followed later by the loss of one of the two genes in the eutherian lineage. This gene loss did not apparently affect collagen mRNAs regulation, suggesting that the single eutherian LARP6 is able to perform a function similar to the two LARP6s ( $a$ and b) of other vertebrates. Eutherian LARP6 and vertebrate LARP6a possess NLS and NES motifs (Supplemental Fig. S4), and human LARP6 was shown to shuttle between the nucleus and the cytoplasm (Valavanis et al. 2007). Also, human LARP6 is likely to bind collagen mRNAs in the nucleus and was proposed to be involved in their nucleocytoplasmic trafficking and translational regulation (Cai et al. 2010a). Based on these observations, one possibility is that in non-eutherian vertebrate LARP6a fulfills the nuclear binding and nucleocytoplasmic trafficking functions but hands over the transcripts to LARP6b, which performs the cytoplasmic regulatory function. The functional characterization of LARP6a and LARP6b will be needed to test this hypothesis.

\section{The distinct RNA-binding properties and subcellular localizations of plant LARP6 support the neofunctionalization scenario upon PAM2 acquisition}

We observed that the reorganization of the plant LARP6 LAM upon PAM2 acquisition was associated with a change in the RNA-binding properties of the proteins (Figs. 7, 8). AtLARP6a can bind in vitro to all tested sequences, but with a clear preference for 20-nt oligo(A) (Fig. 7). Conversely, of the homopolymeric sequences tried, PAM2-containing AtLARP6c binds significantly only to 20-nt oligo(U) (Fig. 8). Although this analysis represents a first step in the full characterization of the RNA-binding properties of the proteins, which, for example, may have affinity for particular RNA sequences and/or structures not investigated here, it is sufficient to delineate that differences in their RNA-binding properties do, indeed, exist.
Further to the RNA-binding profile, the subcellular localization of AtLARP6a compared with AtLARP6b and $c$ is also very different. Whereas AtLARP6a is mainly localized in the nucleolus and does not associate with stress granules (Fig. 9A), AtLARP6b and c are both present in the nuclear compartment, but $6 \mathrm{~b}$ is mainly enriched in the nucleolus, while $6 \mathrm{c}$ is found both in nucleoplasm and nucleolus, and both are found in cytoplasmic stress granules likely to contain mRNAs (Fig. 9B,C). Taken together, these differences in RNA-binding properties and subcellular localization strongly suggest that plant PAM2-containing LARP6s have acquired new functions compared with the "ancestral" plant LARP6 lacking a PAM2 motif.

Finally, while the LARP6b and 6c PAM2 motifs display the same affinity for a canonical PABP MLLE domain in vitro, their respective contributions to the association with the poly(A)-binding protein in the context of the fulllength proteins in vivo is different. The PAM-6b probably fulfills most if not all of the PABP-binding function, while the PAM-6c peptide is likely not the only region of the LARP6c protein to be involved in this binding. As a consequence, we postulate that LARP6c binds to the C-terminal region but also to additional domains of the PABP that could lie across its RRM region, as is the case for Paip1 and Paip2 (Khaleghpour et al. 2001a; Roy et al. 2002). This supports the idea that LARP6b and LARP6c could exert different types of regulation on PABP functions.

The functions of the three plant LARP6 proteins are currently unknown, and further in planta studies are needed to elucidate this. Nevertheless, the preferential localization of AtLARP6a in the nucleolus is intriguing. The nucleolus is a multifunctional nuclear subcompartment whose most prominent and conserved function resides in pre-ribosomal RNA subunits production (for review, see Brown and Shaw 2008). In addition, the nucleolus is a central hub for the biogenesis and TRAMP-mediated quality control of several small noncoding RNPs (Carneiro et al. 2007), the correct mRNP assembly of several mRNAs (at least in yeast and mammals) (Du et al. 2008; Jellbauer and Jansen 2008) and the recognition and tagging to cytoplasmic nonsense-mediated decay of aberrantly spliced pre-mRNAs (at least in plant) (Kim et al. 2009). Whether AtLARP6a may play a role in the noncoding RNA TRAMPmediated surveillance system is not straightforward to predict on the basis of current knowledge. The oligo(A) tails appended at the $3^{\prime}$ ends of the RNAs to be degraded by this process are probably too short (3-5 residues) (Jia et al. 2011, 2012) for the optimal interaction with AtLARP6a, but since this La module shows some affinity to other homopolymers (Fig. 7), we cannot rule out that it could bind to internal sequences as well as to the short oligo(A) tail of TRAMP substrates. Given its presence in both the nucleolus and cytoplasm, another possibility is that AtLARP6a is involved in the nucleolar biogenesis and/or surveillance of mRNPs. 
AtLARP6c and the human LARP4a are both likely to be targeted in vivo to the $3^{\prime}$ UTRs of mRNA following their interaction with the poly(A)-binding protein (see Fig. 6; Yang et al. 2011). Nevertheless, contrary to the latter that binds specifically to oligo(A) sequences, the strong preference of AtLARP6c for U-rich regions is somewhat surprising. The terminal uridylation of mRNAs has been documented and shown to induce decapping and $5^{\prime}-3^{\prime}$ degradation in Schizosaccharomyces pombe and human (Mullen and Marzluff 2008; Rissland and Norbury 2009), and a terminal mRNA uridylyl transferase was also recently characterized in Arabidopsis (Morozov et al. 2012). Nevertheless, we deem it unlikely that AtLARP6c, whose binding to oligo(U) homopolymers is highly length dependent (U20 >> U10) (Fig. 8; Supplemental Fig. S9), intervenes in this mRNA decay and/ or processing mechanism because the terminally added oligo(U) tails are usually short ( $\sim 5 \mathrm{nt}$ long) in vivo and most efficiently stimulate decapping when 5-10 nt long in vitro (Song and Kiledjian 2007). The yeast poly(U)-binding protein (Publp) is a characterized case of an mRNA-binding factor with high and exclusive in vitro specificity for oligo(U) homopolymers (Anderson et al. 1993; Matunis et al. 1993). Publp is both an hnRNP and mRNP factor that relocalizes from nucleus to stress granules upon stress and was shown to protect a large collection of mRNAs from accelerated decay by binding to internal U-rich regions present in their $3^{\prime}$ or $5^{\prime}$ UTRs (Ruiz-Echevarria and Peltz 2000; Duttagupta et al. 2005). It is interesting to note that Publp is a homolog of the mammalian proteins TIA-1/ TIAR known to be an important stress granule component (Gilks et al. 2004) and that AtLARP6c can also be found in stress granules (Fig. 9). Since AtLARP6c binding does not involve $3^{\prime} \mathrm{OH}$ recognition (Supplemental Fig. S9), it may interact with internal U-rich sequences upon its targeting to at least certain mRNAs (by the PAM2-MLLE interaction), thereby influencing their stability and/or translation.

The difficulties encountered in obtaining sufficiently stable and monodisperse recombinant AtLARP6b La modules hampered our in vitro assessment of its RNA-binding abilities. The elaboration of working hypotheses regarding AtLARP6b function in planta is hence extremely limited. Nevertheless, our studies positively identified its ability to bind to the poly(A)-binding protein both in vitro and in vivo and its relocalization to stress granules upon stress, which taken together would suggest that AtLARP6b could somehow be involved in mRNA metabolism.

\section{MATERIALS AND METHODS}

\section{Multiple sequences alignments, phylogenetic reconstruction, and secondary structure predictions}

Sequences were aligned using the multiple sequence comparison by log-expectation (MUSCLE v3.7) software (Edgar 2004). Alignment shading was obtained using BOXSHADE v3.21. Trees were reconstructed using the fast maximum likelihood tree estimation program PHYML (Guindon and Gascuel 2003) with the LG amino acids replacement matrix (Le and Gascuel 2008). Statistical support for the major clusters were obtained using the approximate likelihood-ratio test (aLRT) (Anisimova and Gascuel 2006). The LARP4 and LARP6 RRMs were analyzed with the Hidden-Markov-Model (HMM)-based protein structure prediction program SAM-T08 (http://compbio.soe.ucsc.edu/SAM_T08/ T08-query.html) (Karplus et al. 2005) to identify putative structural segments.

\section{Cloning}

The Arabidopsis AtPAB2 (at4g34110), AtLARP6a (at5g46250), 6b (at2g43970), and 6c (at3g19090) full-length cDNAs were, respectively, PCR-amplified (from total cDNAs prepared from 2-wk-old plantlet RNA) with primer pairs 415/397, 492/493, 495/ 496, or 498/499 (see Supplemental Table S3 for a list of primers used in this study) and subcloned into plasmid pTP1 (a pBluescript derivative) between the AttL1 and AttL2 Gateway recombination sites. To prepare cDNAs expressing LARP $6 \mathrm{~b}$ and $6 \mathrm{c}$ proteins strictly deleted of their PAM2 motifs (amino acids 47-60 for PAM2-6b and 33-46 for PAM2-6c), we proceeded as follows: Fragments of their cDNAs were PCR-amplified from pTP1-LARP6b or pTP1-LARP6c with primers $810 / 811$ or $810 / 815$ to give PCR products $\mathrm{P} 1$ that span from the XhoI site of the vector (upstream of the ATG) to the nucleotide upstream of the PAM2 motif. PCR products P1 were added at their $3^{\prime}$ terminus with a short adaptator corresponding to the $20 \mathrm{nt}$ just downstream from the PAM2 region, by PCR with primers $810 / 814$ (6b) or 810/817 (6c), giving products P2. A third PCR product (P3) spanning from immediately downstream from the PAM2 sequences to the XbaI site of the vector (downstream from the stop codon) was obtained by PCR with primers $812 / 813$ (6b) and 816/813 (6c). The complete $6 \mathrm{~b}$ and $6 \mathrm{c}$ cDNAs deleted of their PAM2 regions were reconstituted by PCR combining fragments P2 and P3 and primers $810 / 813(6 \mathrm{~b}, 6 \mathrm{c})$. The complete fragments were inserted into vector pTP1 at sites XhoI and XbaI.

For recombinant protein production, the fragment of AtPAB2 encoding the MLLE domain (spanning amino acids 539-642) and the La modules from AtLARP6a (region spanning amino acids 78-286) and AtLARP6c (region spanning amino acids 137-332) were, respectively, PCR-amplified with primer pairs RM7/RM8; $608 / 609$ or $659 / 660$ (Supplemental Table S3). The PCR products were subsequently subcloned into the pNEA-tH expression vector (a derivative of pet 15b kindly provided by A.C. DockBregeon, IGBMC, Strasbourg), yielding the pNEA-tHMLLEat, pNEA-tH6amod, and pNEA-tH6cmod vectors.

For subcellular localization and/or coimmunoprecipitation experiments, using the Gateway recombination technique according to the manufacturer's instructions (Invitrogen), the fulllength AtLARP6a, $6 \mathrm{~b}$, and $6 \mathrm{c}$ or $6 \mathrm{~b}-\triangle \mathrm{PAM} 2$ and $6 \mathrm{c}-\Delta \mathrm{PAM} 2$ cDNAs were 5'-OH tagged with the tagRFP, Cerulean (Cer-), or EGFP fluorescent tags under control of the constitutive CaMV 35S promoter using the destination vectors pSITEII-6C1 (tagRFP), pSITEII-2C1 (Cerulean), or pSITEII-3C1 (EGFP) (Martin et al. 2009). A tagRFP-AtPAB2 fusion was also obtained with the pSITEII$6 \mathrm{C} 1$ vector. Every PCR and Gateway recombination product was fully verified by sequencing.

Every insert/fusion, either PCR or Gateway derived, has been comprehensively sequenced. 


\section{Peptide synthesis, recombinant protein production, and Isothermal Titration Calorimetry (ITC) measurements}

To investigate the putative plant PAM2, peptides for ITC experiments were ordered as synthetic HPLC-purified from Genscript (http://www.genscript.com). Their composition and purity were verified by mass spectroscopy. We chose 14-amino-acid-long peptides spanning, respectively, the putative PAM2 (reported in bold) of the Arabidopsis proteins AtLARP6b (PAM6b: residues 47-60: SRLNAGAPEFVPGR) and AtLARP6c (PAM6c: residues 33-46: FKFNAQAPEFVPRS). The His6-tagged AtPAB2 MLLE (593-642) protein was expressed from the pNEA-tHMLLEat plasmid in Escherichia coli Rosetta II cells and purified using affinity Ni-chromatography followed by ion-exchange (DEAE). The purified protein was dialyzed in a buffer containing $50 \mathrm{mM}$ phosphate, $150 \mathrm{mM} \mathrm{NaCl}, 1 \mathrm{mM}$ DTT (pH 6.3) and used for ITC experiments with the PAM2 peptides. The calorimetric analysis was carried out in the same experimental conditions as reported by Siddiqui et al. (2007), i.e., $50 \mathrm{mM}$ phosphate, $150 \mathrm{mM} \mathrm{NaCl}$, $1 \mathrm{mM}$ DTT $(\mathrm{pH} 6.3)$ at $15^{\circ} \mathrm{C}$, using an ITC-200 microcalorimeter from Microcal (GE Healthcare). In each titration, volumes of $2 \mu \mathrm{L}$ of a $180 \mu \mathrm{M}$ solution of AtPAB2 MLLE were injected into a solution of PAM6b or PAM6c $(20 \mu \mathrm{M})$, using a computer-controlled $40-\mu \mathrm{L}$ microsyringe. To allow the system to reach equilibrium, a spacing of $180 \mathrm{sec}$ between each injection was applied. Integrated heat data obtained for the titrations were fitted using a nonlinear least-squares minimization algorithm to a theoretical titration curve, using the MicroCal-Origin 7.0 software package. $\Delta H^{\circ}$ (reaction enthalpy change in kilocalories per mole), $\mathrm{K}_{\mathrm{b}}$ (binding constant in per mole), and $n$ (molar ratio between the two proteins in the complex) were the fitting parameters. The reaction entropy was calculated using the relationships $\Delta G=$ $-R T \cdot \ln K_{\mathrm{b}}(\mathrm{R}=1.986 \mathrm{cal} / \mathrm{mol} \cdot \mathrm{K}, T=298 \mathrm{~K})$ and $\Delta G=\Delta H-T$ $\Delta S$. All ITC experiments were repeated at least three times, and a detailed analysis of the thermodynamic parameters and errors calculated as the standard deviation from the mean value are reported in Supplemental Table S2.

AtLARP6a and AtLARP6c La modules (LAM-RRM) were expressed from plasmids pNEA-tH6amod or pNEA-tH6cmod in E. coli Rosetta II cells at $37^{\circ} \mathrm{C}$ and $18^{\circ} \mathrm{C}$, respectively. Cell pellets were lysed by sonication in $50 \mathrm{mM}$ Tris, $300 \mathrm{mM} \mathrm{NaCl}$, $10 \mathrm{mM}$ imidazole, 5\% glycerol ( $\mathrm{pH} 8.0$ ), with $2 \mathrm{mM}$ PMSF (phenylmethanesulfonyl fluoride) and lysozyme. Lysates were clarified by centrifugation and applied onto 5-mL HisTrap columns (GE Healthcare). Fractions containing the eluted protein were then loaded onto a 5-mL Hi-Trap heparin column, mainly to eliminate nucleic acid contaminants. The protein solution was dialyzed in $20 \mathrm{mM}$ Tris, $100 \mathrm{mM} \mathrm{KCl}, 0.2 \mathrm{mM}$ EDTA, $1 \mathrm{mM}$ DTT (pH 7.25) (for NMR) and $20 \mathrm{mM}$ Tris, $200 \mathrm{mM} \mathrm{KCl}, 0.2 \mathrm{mM}$ EDTA, 1 mM DTT (pH 7.25) (for ITC). Heat produced by RNA dilution was verified to be negligible by performing control titrations into the buffer alone, under the same conditions. All ITC experiments were repeated at least three times; the thermodynamic parameters are reported in Supplemental Table S2. Protein concentrations were calculated based on the near-UV absorption $\left(\varepsilon_{280}\right)$ using theoretical extinction coefficients derived from ExPASY.

To study the RNA-binding properties of the LARP6 protein La modules, RNA oligonucleotides were purchased from IBA-GmBH (http://www.iba-go.com). The ITC experiments were performed in $20 \mathrm{mM}$ Tris, $200 \mathrm{mM} \mathrm{KCl}, 0.2 \mathrm{mM}$ EDTA, $1 \mathrm{mM}$ DTT (pH 7.25 ) at $298 \mathrm{~K}$ using a Microcal ITC-200 microcalorimeter as previously described (Hands-Taylor et al. 2010). In each titration, 20 injections of $2 \mu \mathrm{L}$ each of a RNA solution, at a concentration of $200 \mu \mathrm{M}$, were added into a $20 \mu \mathrm{M}$ protein solution, using a computer-controlled $40-\mu \mathrm{L}$ microsyringe. Data fitting was performed as described above.

\section{Immunoprecipitation experiments}

$N$. benthamiana leaves were co-agroinfiltrated as previously described (Grefen et al. 2010) with an equimolar mix of a suspension of Agrobacterium tumefaciens (GV3101 strain) carrying the pSITEtagRFP-PAB2 plasmid and a suspension of A. tumefaciens carrying the pSITE-EGFP-AtLARP6a, the pSITE-EGFP-AtLARP6b, or the pSITE-EGFP-AtLARP6c vector. Agro-infiltrated leaves were harvested and immediately frozen into liquid nitrogen $48 \mathrm{~h}$ after co-infiltration. To perform coimmunoprecipitation experiments, a crude extract was prepared as follows: $200 \mathrm{mg}$ of liquid nitrogen pulverized leaves were homogenized into $1 \mathrm{~mL}$ of ice-cold lysis buffer $\left(50 \mathrm{mM}\right.$ Tris- $\mathrm{HCl}$ at $\mathrm{pH} 7.5,5 \mathrm{mM} \mathrm{MgCl}_{2}, 150 \mathrm{mM} \mathrm{NaCl}$, $10 \%$ glycerol, $1 \%$ NP-40, $2 \%$ plant protease inhibitor cocktail [Sigma-Aldrich P9599]) and clarified twice by $15 \mathrm{~min}$ of centrifugation $\left(16,000 \mathrm{~g}\right.$ at $\left.4^{\circ} \mathrm{C}\right)$. To perform the immunoprecipitation, $750 \mu \mathrm{L}$ of crude extract was used with anti-GFP using the $\mu$ MACS GFP isolation kit from Miltenyi Biotech according to the manufacturer's instructions. The input and eluate fractions were separated by SDS-PAGE electrophoresis, transferred to a nitrocellulose membrane, and blotted with anti-PAB2 (Dufresne et al. 2008) antibodies diluted at $1 / 7500$. After stripping, the membrane was blotted with monoclonal anti-GFP antibodies (Clontech) diluted at $1 / 2000$.

To quantify the signals obtained by Western blot analysis, we used a Chemidoc (Bio-Rad). Briefly, we acquired pictures every $1 \mathrm{sec}$ until appearance of the first saturating signal. Quantification was subsequently performed on the last picture taken before the saturating point.

\section{Subcellular localization experiments}

Onion epidermal cells were transiently transformed using a Biolistic PDS-1000/He Particle delivery system (Bio-Rad) according to the manufacturer's instructions. Onion cells were cotransformed either with pSITE-tagRFP-LARP6a, pSITE-tagRFP-LARP6b, or pSITE-tagRFP-LARP6c and pGFP-NOP10 (gift from J. Rodor, M. Echeverria, LGDP, Perpignan) vectors or cotransformed with pSITE-tagRFP-AtPAB2 plus pSITE-Cer-LARP6a, 6b, or $6 \mathrm{c}$ plasmids for colocalization analysis. Sixteen to $24 \mathrm{~h}$ after bombardment, the onion epidermis were peeled off and analyzed with a LSM710 Zeiss confocal microscope. Two to $4 \mathrm{~h}$ after placing the onion epidermis on slide, a hypoxia-derived stress state was monitored following the aggregation of the AtPAB2 protein into subcytoplasmic granules (stress granules) as previously reported (Weber et al. 2008).

\section{SUPPLEMENTAL MATERIAL}

Supplemental material is available for this article. 


\section{ACKNOWLEDGMENTS}

M.R.C. is indebted to the Wellcome Trust for the Centre of Biomolecular Spectroscopy. L.M. is a long-term EMBO fellow. R.M. was the recipient of a Universite de Perpignan (UPVD) short-term fellowship followed by an ANR fellowship. This work was supported by l'Agence National pour la Recherche (ANR-2010 BLAN 1707 01), by the CNRS and the UPVD through utilization of the Chemidoc and confocal microscope at the Technoviv Plateform. We thank Dr. A.C. Dock-Bregeon (IGBMC, Strasbourg) for the gift of the pNEAtH E. coli expression vector and Dr. M. Goodin (University of Calgary, Canada) for the gift of the pSITE vector series. We are extremely grateful to Dr. J.F. Laliberté (INRS, Quebec, Canada) for his generous gift of antiPAB2 antibodies.

Received July 11, 2012; accepted October 12, 2012.

\section{REFERENCES}

Anderson P, Kedersha N. 2009. RNA granules: Post-transcriptional and epigenetic modulators of gene expression. Nat Rev Mol Cell Biol 10: 430-436.

Anderson JT, Paddy MR, Swanson MS. 1993. PUB1 is a major nuclear and cytoplasmic polyadenylated RNA-binding protein in Saccharomyces cerevisiae. Mol Cell Biol 13: 6102-6113.

Anisimova M, Gascuel O. 2006. Approximate likelihood-ratio test for branches: A fast, accurate, and powerful alternative. Syst Biol 55: 539-552.

Bayfield MA, Maraia RJ. 2009. Precursor-product discrimination by La protein during tRNA metabolism. Nat Struct Mol Biol 16: 430437.

Bayfield MA, Yang R, Maraia RJ. 2010. Conserved and divergent features of the structure and function of La and La-related proteins (LARPs). Biochim Biophys Acta 1799: 365-378.

Blagden SP, Gatt MK, Archambault V, Lada K, Ichihara K, Lilley KS, Inoue YH, Glover DM. 2009. Drosophila Larp associates with poly(A)-binding protein and is required for male fertility and syncytial embryo development. Dev Biol 334: 186-197.

Bousquet-Antonelli C, Deragon JM. 2009. A comprehensive analysis of the La-motif protein superfamily. RNA 15: 750-764.

Brown JW, Shaw PJ. 2008. The role of the plant nucleolus in premRNA processing. Curr Top Microbiol Immunol 326: 291-311.

Burrows C, Abd Latip N, Lam SJ, Carpenter L, Sawicka K, Tzolovsky G, Gabra H, Bushell M, Glover DM, Willis AE, et al. 2010. The RNA binding protein Larp1 regulates cell division, apoptosis and cell migration. Nucleic Acids Res 38: 5542-5553.

Cai L, Fritz D, Stefanovic L, Stefanovic B. 2010a. Binding of LARP6 to the conserved $5^{\prime}$ stem-loop regulates translation of mRNAs encoding type I collagen. J Mol Biol 395: 309-326.

Cai L, Fritz D, Stefanovic L, Stefanovic B. 2010b. Nonmuscle myosindependent synthesis of type I collagen. J Mol Biol 401: 564-578.

Carneiro T, Carvalho C, Braga J, Rino J, Milligan L, Tollervey D, Carmo-Fonseca M. 2007. Depletion of the yeast nuclear exosome subunit Rrp6 results in accumulation of polyadenylated RNAs in a discrete domain within the nucleolus. Mol Cell Biol 27: 4157-4165.

Clery A, Blatter M, Allain FH. 2008. RNA recognition motifs: Boring? Not quite. Curr Opin Struct Biol 18: 290-298.

Craig AW, Haghighat A, Yu AT, Sonenberg N. 1998. Interaction of polyadenylate-binding protein with the eIF4G homologue PAIP enhances translation. Nature 392: 520-523.

Diribarne G, Bensaude O. 2009. 7SK RNA, a non-coding RNA regulating P-TEFb, a general transcription factor. RNA Biol 6: 122-128.

Dong G, Chakshusmathi G, Wolin SL, Reinisch KM. 2004. Structure of the La motif: A winged helix domain mediates RNA binding via a conserved aromatic patch. EMBO J 23: 1000-1007.
Du TG, Jellbauer S, Muller M, Schmid M, Niessing D, Jansen RP. 2008. Nuclear transit of the RNA-binding protein She 2 is required for translational control of localized ASH1 mRNA. EMBO Rep 9: 781-787.

Dufresne PJ, Ubalijoro E, Fortin MG, Laliberte JF. 2008. Arabidopsis thaliana class II poly(A)-binding proteins are required for efficient multiplication of turnip mosaic virus. J Gen Virol 89: 2339-2348.

Duttagupta R, Tian B, Wilusz CJ, Khounh DT, Soteropoulos P, Ouyang M, Dougherty JP, Peltz SW. 2005. Global analysis of Publp targets reveals a coordinate control of gene expression through modulation of binding and stability. Mol Cell Biol 25: 5499-5513.

Edgar RC. 2004. MUSCLE: A multiple sequence alignment method with reduced time and space complexity. BMC Bioinformatics 5: 113. doi: 10.1186/1471-2105-5-113.

Funakoshi Y, Doi Y, Hosoda N, Uchida N, Osawa M, Shimada I, Tsujimoto M, Suzuki T, Katada T, Hoshino S. 2007. Mechanism of mRNA deadenylation: Evidence for a molecular interplay between translation termination factor eRF3 and mRNA deadenylases. Genes Dev 21: 3135-3148.

Gilks N, Kedersha N, Ayodele M, Shen L, Stoecklin G, Dember LM, Anderson P. 2004. Stress granule assembly is mediated by prionlike aggregation of TIA-1. Mol Biol Cell 15: 5383-5398.

Grefen C, Donald N, Hashimoto K, Kudla J, Schumacher K, Blatt MR. 2010. A ubiquitin-10 promoter-based vector set for fluorescent protein tagging facilitates temporal stability and native protein distribution in transient and stable expression studies. Plant $J$ 64: $355-365$.

Guindon S, Gascuel O. 2003. A simple, fast, and accurate algorithm to estimate large phylogenies by maximum likelihood. Syst Biol 52: 696-704.

Hands-Taylor KL, Martino L, Tata R, Babon JJ, Bui TT, Drake AF, Beavil RL, Pruijn GJ, Brown PR, Conte MR. 2010. Heterodimerization of the human RNase P/MRP subunits Rpp20 and Rpp25 is a prerequisite for interaction with the P3 arm of RNase MRP RNA. Nucleic Acids Res 38: 4052-4066.

He X, Zhang J. 2005. Rapid subfunctionalization accompanied by prolonged and substantial neofunctionalization in duplicate gene evolution. Genetics 169: 1157-1164.

Holcik M, Sonenberg N. 2005. Translational control in stress and apoptosis. Nat Rev Mol Cell Biol 6: 318-327.

Jellbauer S, Jansen RP. 2008. A putative function of the nucleolus in the assembly or maturation of specialized messenger ribonucleoprotein complexes. RNA Biol 5: 225-229.

Jia H, Wang X, Liu F, Guenther UP, Srinivasan S, Anderson JT, Jankowsky E. 2011. The RNA helicase Mtr4p modulates polyadenylation in the TRAMP complex. Cell 145: 890-901.

Jia H, Wang X, Anderson JT, Jankowsky E. 2012. RNA unwinding by the Trf4/Air2/Mtr4 polyadenylation (TRAMP) complex. Proc Natl Acad Sci 109: 7292-7297.

Karplus K, Katzman S, Shackleford G, Koeva M, Draper J, Barnes B, Soriano M, Hughey R. 2005. SAM-T04: What is new in protein-structure prediction for CASP6. Proteins (Suppl 7) 61: 135-142.

Katzenellenbogen RA, Egelkrout EM, Vliet-Gregg P, Gewin LC, Gafken PR, Galloway DA. 2007. NFX1-123 and poly(A) binding proteins synergistically augment activation of telomerase in human papillomavirus type 16 E6-expressing cells. J Virol 81: 3786-3796.

Khaleghpour K, Kahvejian A, De Crescenzo G, Roy G, Svitkin YV, Imataka H, O'Connor-McCourt M, Sonenberg N. 2001a. Dual interactions of the translational repressor Paip2 with poly(A) binding protein. Mol Cell Biol 21: 5200-5213.

Khaleghpour K, Svitkin YV, Craig AW, DeMaria CT, Deo RC, Burley SK, Sonenberg N. 2001b. Translational repression by a novel partner of human poly(A) binding protein, Paip2. Mol Cell 7: 205-216.

Kim SH, Koroleva OA, Lewandowska D, Pendle AF, Clark GP, Simpson CG, Shaw PJ, Brown JW. 2009. Aberrant mRNA 
transcripts and the nonsense-mediated decay proteins UPF2 and UPF3 are enriched in the Arabidopsis nucleolus. Plant Cell 21: 2045-2057.

Kotik-Kogan O, Valentine ER, Sanfelice D, Conte MR, Curry S. 2008. Structural analysis reveals conformational plasticity in the recognition of RNA $3^{\prime}$ ends by the human La protein. Structure 16: $852-$ 862.

Kozlov G, Menade M, Rosenauer A, Nguyen L, Gehring K. 2010. Molecular determinants of PAM2 recognition by the MLLE domain of poly(A)-binding protein. J Mol Biol 397: 397-407.

Le SQ, Gascuel O. 2008. An improved general amino acid replacement matrix. Mol Biol Evol 25: 1307-1320.

Lim NS, Kozlov G, Chang TC, Groover O, Siddiqui N, Volpon L, De Crescenzo G, Shyu AB, Gehring K. 2006. Comparative peptide binding studies of the PABC domains from the ubiquitin-protein isopeptide ligase $\mathrm{HYD}$ and poly(A)-binding protein. Implications for HYD function. J Biol Chem 281: 14376-14382.

Maris C, Dominguez C, Allain FH. 2005. The RNA recognition motif, a plastic RNA-binding platform to regulate post-transcriptional gene expression. FEBS J 272: 2118-2131.

Martin K, Kopperud K, Chakrabarty R, Banerjee R, Brooks R, Goodin MM. 2009. Transient expression in Nicotiana benthamiana fluorescent marker lines provides enhanced definition of protein localization, movement and interactions in planta. Plant $J$ 59: $150-162$.

Martineau Y, Derry MC, Wang X, Yanagiya A, Berlanga JJ, Shyu AB, Imataka H, Gehring K, Sonenberg N. 2008. Poly(A)-binding protein-interacting protein 1 binds to eukaryotic translation initiation factor 3 to stimulate translation. Mol Cell Biol 28: 6658-6667.

Martino L, Pennell S, Kelly G, Bui TT, Kotik-Kogan O, Smerdon SJ, Drake AF, Curry S, Conte MR. 2012. Analysis of the interaction with the hepatitis $\mathrm{C}$ virus mRNA reveals an alternative mode of RNA recognition by the human La protein. Nucleic Acids Res 40: 1381-1394.

Matunis MJ, Matunis EL, Dreyfuss G. 1993. PUB1: A major yeast poly(A) ${ }^{+}$RNA-binding protein. Mol Cell Biol 13: 6114-6123.

Merzlyak EM, Goedhart J, Shcherbo D, Bulina ME, Shcheglov AS, Fradkov AF, Gaintzeva A, Lukyanov KA, Lukyanov S, Gadella TW, et al. 2007. Bright monomeric red fluorescent protein with an extended fluorescence lifetime. Nat Methods 4: 555-557.

Morozov IY, Jones MG, Gould PD, Crome V, Wilson JB, Hall AJ, Rigden DJ, Caddick MX. 2012. mRNA 3'-tagging is induced by nonsense mediated decay and promotes ribosome dissociation. Mol Cell Biol 13: 6114-6123.

Mullen TE, Marzluff WF. 2008. Degradation of histone mRNA requires oligouridylation followed by decapping and simultaneous degradation of the mRNA both $5^{\prime}$ to $3^{\prime}$ and $3^{\prime}$ to $5^{\prime}$. Genes Dev 22: $50-65$.

Nykamp K, Lee MH, Kimble J. 2008. C. elegans La-related protein, LARP-1, localizes to germline $\mathrm{P}$ bodies and attenuates Ras-MAPK signaling during oogenesis. RNA 14: 1378-1389.

Okochi K, Suzuki T, Inoue J, Matsuda S, Yamamoto T. 2005. Interaction of anti-proliferative protein Tob with poly(A)-binding protein and inducible poly(A)-binding protein: Implication of Tob in translational control. Genes Cells 10: 151-163.

Palanivelu R, Belostotsky DA, Meagher RB. 2000a. Arabidopsis thaliana poly (A) binding protein 2 (PAB2) functions in yeast translational and mRNA decay processes. Plant J 22: 187-198.

Palanivelu R, Belostotsky DA, Meagher RB. 2000b. Conserved expression of Arabidopsis thaliana poly(A) binding protein 2
(PAB2) in distinct vegetative and reproductive tissues. Plant $J$ 22: 199-210.

Parsons CJ, Stefanovic B, Seki E, Aoyama T, Latour AM, Marzluff WF, Rippe RA, Brenner DA. 2011. Mutation of the $5^{\prime}$-untranslated region stem-loop structure inhibits $\alpha 1$ (I) collagen expression in vivo. J Biol Chem 286: 8609-8619.

Rissland OS, Norbury CJ. 2009. Decapping is preceded by 3' uridylation in a novel pathway of bulk mRNA turnover. Nat Struct Mol Biol 16: 616-623.

Roy G, De Crescenzo G, Khaleghpour K, Kahvejian A, O'ConnorMcCourt M, Sonenberg N. 2002. Paipl interacts with poly(A) binding protein through two independent binding motifs. Mol Cell Biol 22: 3769-3782.

Ruiz-Echevarria MJ, Peltz SW. 2000. The RNA binding protein Pub1 modulates the stability of transcripts containing upstream open reading frames. Cell 101: 741-751.

Schaffler K, Schulz K, Hirmer A, Wiesner J, Grimm M, Sickmann A, Fischer U. 2010. A stimulatory role for the La-related protein $4 \mathrm{~B}$ in translation. RNA 16: 1488-1499.

Siddiqui N, Osborne MJ, Gallie DR, Gehring K. 2007. Solution structure of the PABC domain from wheat poly(A)-binding protein: An insight into RNA metabolic and translational control in plants. Biochemistry 46: 4221-4231.

Singh M, Wang Z, Koo BK, Patel A, Cascio D, Collins K, Feigon J. 2012. Structural basis for telomerase RNA recognition and RNP assembly by the holoenzyme La family protein p65. Mol Cell 47: $16-26$.

Song MG, Kiledjian M. 2007. 3' terminal oligo U-tract-mediated stimulation of decapping. RNA 13: 2356-2365.

Svitkin YV, Evdokimova VM, Brasey A, Pestova TV, Fantus D, Yanagiya A, Imataka $\mathrm{H}$, Skabkin MA, Ovchinnikov LP, Merrick WC, et al. 2009. General RNA-binding proteins have a function in poly(A)-binding protein-dependent translation. EMBO J 28: 58-68.

Teplova M, Yuan YR, Phan AT, Malinina L, Ilin S, Teplov A, Patel DJ. 2006. Structural basis for recognition and sequestration of $\mathrm{UUU}(\mathrm{OH}) 3^{\prime}$ temini of nascent RNA polymerase III transcripts by La, a rheumatic disease autoantigen. Mol Cell 21: 75-85.

Uchida N, Hoshino S, Imataka H, Sonenberg N, Katada T. 2002. A novel role of the mammalian GSPT/eRF3 associating with poly(A)-binding protein in Cap/poly(A)-dependent translation. J Biol Chem 277: 50286-50292.

Valavanis C, Wang Z, Sun D, Vaine M, Schwartz LM. 2007. Acheron, a novel member of the Lupus Antigen family, is induced during the programmed cell death of skeletal muscles in the moth Manduca sexta. Gene 393: 101-109.

Weber C, Nover L, Fauth M. 2008. Plant stress granules and mRNA processing bodies are distinct from heat stress granules. Plant J 56: 517-530.

Witkin KL, Collins K. 2004. Holoenzyme proteins required for the physiological assembly and activity of telomerase. Genes Dev 18: $1107-1118$

Wolin SL, Cedervall T. 2002. The La protein. Annu Rev Biochem 71: 375-403.

Xue Y, Yang Z, Chen R, Zhou Q. 2010. A capping-independent function of MePCE in stabilizing 7SK snRNA and facilitating the assembly of 7SK snRNP. Nucleic Acids Res 38: 360-369.

Yang R, Gaidamakov SA, Xie J, Lee J, Martino L, Kozlov G, Crawford AK, Russo AN, Conte MR, Gehring K, et al. 2011. La-related protein 4 binds poly(A), interacts with the poly(A)-binding protein MLLE domain via a variant PAM2 $w$ motif, and can promote mRNA stability. Mol Cell Biol 31: 542-556. 

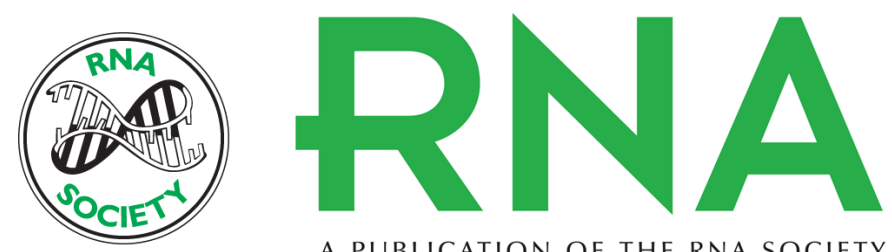

A PUBLICATION OF THE RNA SOCIETY

\section{The association of a La module with the PABP-interacting motif PAM2 is a recurrent evolutionary process that led to the neofunctionalization of La-related proteins}

Rémy Merret, Luigi Martino, Cécile Bousquet-Antonelli, et al.

RNA 2013 19: 36-50 originally published online November 12, 2012

Access the most recent version at doi:10.1261/rna.035469.112

Supplemental Material

References

Open Access

License Freely available online through the RNA Open Access option.

Email Alerting Service

Freely available online through the RNA Open Access option. top right corner of the article or click here.
http://rnajournal.cshlp.org/content/suppl/2012/11/06/rna.035469.112.DC1

This article cites 68 articles, 22 of which can be accessed free at: http://rnajournal.cshlp.org/content/19/1/36.full.html\#ref-list-1

Receive free email alerts when new articles cite this article - sign up in the box at the 